TO APPEAR IN The Astrophysical Journal.

Preprint typeset using LATEX style emulateapj v. 5/2/11

\title{
SUPERMASSIVE BLACK HOLES WITH HIGH ACCRETION RATES IN ACTIVE GALACTIC NUCLEI. V. A NEW SIZE-LUMINOSITY SCALING RELATION FOR THE BROAD-LINE REGION
}

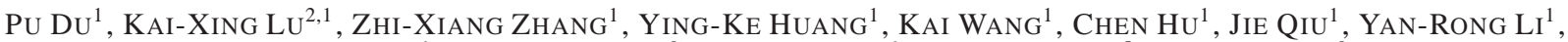

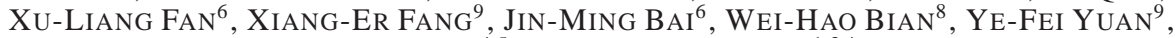 \\ LUIS C. Ho ${ }^{4,5}$ AND JIAN-MIN WANG ${ }^{1,3, *}$ \\ (SEAMBH COLLABORATION) \\ Received 2015 December 13; accepted 2016 April 20
}

\begin{abstract}
This paper reports results of the third-year campaign of monitoring super-Eddington accreting massive black holes (SEAMBHs) in active galactic nuclei (AGNs) between 2014-2015. Ten new targets were selected from quasar sample of Sloan Digital Sky Survey (SDSS), which are generally more luminous than the SEAMBH candidates in last two years. $\mathrm{H} \beta$ lags $\left(\tau_{\mathrm{H} \beta}\right)$ in five of the 10 quasars have been successfully measured in this monitoring season. We find that the lags are generally shorter, by large factors, than those of objects with same optical luminosity, in light of the well-known $R_{\mathrm{H} \beta}-L_{5100}$ relation. The five quasars have dimensionless accretion rates of $\dot{\mathscr{M}}=10-10^{3}$. Combining measurements of the previous SEAMBHs, we find that the reduction of $\mathrm{H} \beta$ lags tightly depends on accretion rates, $\tau_{\mathrm{H} \beta} / \tau_{R-L} \propto \dot{\mathscr{M}}^{-0.42}$, where $\tau_{R-L}$ is the $\mathrm{H} \beta$ lag from the normal $R_{\mathrm{H} \beta}-L_{5100}$ relation. Fitting 63 mapped AGNs, we present a new scaling relation for the broad-line region: $R_{\mathrm{H} \beta}=\alpha_{1} \ell_{44}^{\beta_{1}} \min \left[1,\left(\dot{\mathscr{M}} / \dot{\mathscr{M}}_{c}\right)^{-\gamma_{1}}\right]$, where $\ell_{44}=L_{5100} / 10^{44} \mathrm{erg} \mathrm{s}^{-1}$ is $5100 \AA$ continuum luminosity, and coefficients of $\alpha_{1}=\left(29.6_{-2.8}^{+2.7}\right) \mathrm{lt}-\mathrm{d}, \beta_{1}=0.56_{-0.03}^{+0.03}, \gamma_{1}=0.52_{-0.16}^{+0.33}$ and $\dot{\mathscr{M}}_{c}=11.19_{-6.22}^{+2.29}$. This relation is applicable to AGNs over a wide range of accretion rates, from $10^{-3}$ to $10^{3}$. Implications of this new relation are briefly discussed.

Subject headings: black holes: accretion - galaxies: active - galaxies: nuclei
\end{abstract}

\section{INTRODUCTION}

This is the fifth paper of the series reporting the ongoing large campaign of monitoring Super-Eddington Accreting Massive Black Holes (SEAMBHs) in active galaxies and quasars starting from October 2012. One of the major goals of the campaign is to search for massive black holes with extreme accretion rates through reverberation mapping (RM) of broad emission lines and continuum. Results from the campaigns in 2012-2013 and 2013-2014 have been reported by Du et al. (2014, 2015, hereafter Papers I and IV), Wang et al. (2014, Paper II) and Hu et al. (2015, Paper III). This paper carries out the results of SEAMBH2014 sample, which was monitored from September 2014 to June 2015. With the three monitoring years of observations, we build up a new scaling relation of the broad-line region (BLR) in this paper.

\footnotetext{
${ }^{1}$ Key Laboratory for Particle Astrophysics, Institute of High Energy Physics, Chinese Academy of Sciences, 19B Yuquan Road, Beijing 100049, China

${ }^{2}$ Astronomy Department, Beijing Normal University, Beijing 100875, China

${ }^{3}$ National Astronomical Observatories of China, Chinese Academy of Sciences, 20A Datun Road, Beijing 100020, China

${ }^{4}$ Kavli Institute for Astronomy and Astrophysics, Peking University, Beijing 100871, China

${ }^{5}$ Department of Astronomy, School of Physics, Peking University, Beijing 100871, China

${ }^{6}$ Yunnan Observatories, Chinese Academy of Sciences, Kunming 650011, China

${ }^{8}$ Physics Department, Nanjing Normal University, Nanjing 210097, China

${ }^{9}$ Department of Astronomy, University of Science and Technology of China, Hefei 230026, China

* Corresponding author; wangjm@ihep.ac.cn
}

Reverberation mapping ( $\mathrm{RM}$ ) technique, measuring the delayed echoes of broad lines to the varying ionizing continuum (Bahcall et al. 1972; Blandford \& McKee 1982; Peterson 1993), is a powerful tool to probe the kinematics and geometry of the BLRs in the time domain. Countless clouds, which contribute to the smooth profiles of the broad emission lines (e.g., Arav et al. 1997), are distributed in the vicinity of supermassive black hole (SMBH), composing the BLR. As an observational consequence of photonionization powered by the accretion disk under the deep gravitational potential of the $\mathrm{SMBH}$, the profiles of the lines are broadened, and line emission from the clouds reverberate in response to the varying ionizing continuum. The reverberation is delayed because of light travel difference between $\mathrm{H} \beta$ and ionizing photons and is thus expected to deliver information on the kinematics and structure of the BLR. The unambiguous reverberation of the lines, detected by monitoring campaigns from ultraviolet to optical bands since the late 1980s, supports this picture of the central engine of AGNs (e.g., Clavel et al. 1991; Peterson et al. 1991, 1993; Maoz et al. 1991; Wanders et al. 1993; Dietrich et al. 1993, 1998, 2012; Kaspi et al. 2000; Denney et al. 2006, 2010; Bentz et al. 2009, 2014; Grier et al. 2012; Papers I-IV; Barth et al. 2013, 2015; Shen et al. 2015a,b). The $R_{\mathrm{H} \beta}-L_{5100}$ relation was first discussed by Koratkar \& Gaskell (1991) and Peterson (1993). Robust RM results for 41 AGNs in the last four decades lead to a simple, highly significant correlation of the form

$$
R_{\mathrm{H} \beta} \approx \alpha_{0} \ell_{44}^{\beta_{0}},
$$

where $\ell_{44}=L_{5100} / 10^{44} \mathrm{erg} \mathrm{s}^{-1}$ is the $5100 \AA$ luminosity in units of $10^{44} \mathrm{erg} \mathrm{s}^{-1}$ (corrected for host galaxy contamination) and $R_{\mathrm{H} \beta}=c \tau_{\mathrm{H} \beta}$ is the emissivity-weighted radius of the BLR (Kaspi et al. 2000; Bentz et al. 2013). We refer to this type 
Table 1

The SEAMBH project: observational results

\begin{tabular}{|c|c|c|c|c|c|c|c|}
\hline \multirow[t]{2}{*}{ Object } & \multirow{2}{*}{$\alpha_{2000}$} & \multirow[t]{2}{*}{$\delta_{2000}$} & \multirow[t]{2}{*}{ redshift } & \multirow[t]{2}{*}{ monitoring period } & \multirow[t]{2}{*}{$N_{\text {spec }}$} & \multicolumn{2}{|c|}{ Comparison stars } \\
\hline & & & & & & $R_{*}$ & P.A. \\
\hline \multicolumn{8}{|c|}{ First phase: SEAMBH2012 sample } \\
\hline Mrk 335 & 000619.5 & +201210 & 0.0258 & Oct., 2012 - Feb., 2013 & 91 & $80^{\prime \prime} .7$ & $174.5^{\circ}$ \\
\hline Mrk 1044 & 023005.5 & -085953 & 0.0165 & Oct., 2012 - Feb., 2013 & 77 & $207^{\prime \prime} .0$ & $-143.0^{\circ}$ \\
\hline IRAS $04416+1215$ & 044428.8 & +122112 & 0.0889 & Oct., 2012 - Mar., 2013 & 92 & $137^{\prime \prime} .9$ & $-55.0^{\circ}$ \\
\hline Mrk 382 & 075525.3 & +391110 & 0.0337 & Oct., 2012 - May., 2013 & 123 & $198^{\prime \prime} .4$ & $-24.6^{\circ}$ \\
\hline Mrk 142 & 102531.3 & +514035 & 0.0449 & Nov., 2012 - Apr., 2013 & 119 & $113^{\prime \prime} .1$ & $155.2^{\circ}$ \\
\hline $\mathrm{MCG}+06-26-012$ & 113913.9 & +335551 & 0.0328 & Jan., 2013 - Jun., 2013 & 34 & $204^{\prime \prime} .3$ & $46.1^{\circ}$ \\
\hline IRAS F12397+3333 & 124210.6 & +331703 & 0.0435 & Jan., 2013 - May., 2013 & 51 & $189^{\prime \prime} .0$ & $130.0^{\circ}$ \\
\hline Mrk 486 & 153638.3 & +543333 & 0.0389 & Mar., 2013 - Jul., 2013 & 45 & $193^{\prime \prime} .8$ & $-167.0^{\circ}$ \\
\hline Mrk 493 & 155909.6 & +350147 & 0.0313 & Apr., 2013 - Jun., 2013 & 27 & $155^{\prime \prime} .3$ & $98.5^{\circ}$ \\
\hline \multicolumn{8}{|c|}{ Second phase: SEAMBH2013 sample } \\
\hline$\overline{\text { SDSS J075101.42+291419.1 }}$ & 075101.4 & +291419 & 0.1208 & Nov., 2013 - May., 2014 & 38 & $133^{\prime \prime} .3$ & $-41.3^{\circ}$ \\
\hline SDSS J080101.41+184840.7 & 080101.4 & +184840 & 0.1396 & Nov., 2013 - Apr., 2014 & 34 & $118^{\prime \prime} .8$ & $-98.2^{\circ}$ \\
\hline SDSS J080131.58+354436.4 & 080131.6 & +354436 & 0.1786 & Nov., 2013 - Apr., 2014 & 31 & $100^{\prime \prime} .0$ & $145.2^{\circ}$ \\
\hline SDSS J081441.91+212918.5 & 081441.9 & +212919 & 0.1628 & Nov., 2013 - May., 2014 & 34 & $79^{\prime \prime} .0$ & $73.9^{\circ}$ \\
\hline SDSS J081456.10+532533.5 & 081456.1 & +532534 & 0.1197 & Nov., 2013 - Apr., 2014 & 27 & $164^{\prime \prime} .5$ & $-172.9^{\circ}$ \\
\hline SDSS J093922.89+370943.9 & 093922.9 & +370944 & 0.1859 & Nov., 2013 - Jun., 2014 & 26 & $175^{\prime \prime} .1$ & $-139.0^{\circ}$ \\
\hline \multicolumn{8}{|c|}{ Third phase: SEAMBH2014 sample } \\
\hline SDSS J075949.54+320023.8 & 075949.5 & +320024 & 0.1880 & Sep., 2014 - May., 2015 & 27 & $109^{\prime \prime} .2$ & $-48.3^{\circ}$ \\
\hline SDSS J080131.58+354436.4 & 080131.6 & +354436 & 0.1786 & Oct., 2014 - May., 2015 & 19 & $139^{\prime \prime} .2$ & $-85.3^{\circ}$ \\
\hline SDSS J084533.28+474934.5 & 084533.3 & +474935 & 0.3018 & Sep., 2014 - Apr., 2015 & 18 & $205^{\prime \prime} .5$ & $-126.4^{\circ}$ \\
\hline SDSS J085946.35+274534.8 & 085946.4 & +274535 & 0.2438 & Sep., 2014 - Jun., 2015 & 26 & $169^{\prime \prime} .8$ & $-89.1^{\circ}$ \\
\hline SDSS J102339.64+523349.6 & 102339.6 & +523350 & 0.1364 & Oct., 2014 - Jun., 2015 & 26 & $123^{\prime \prime} .2$ & $108.1^{\circ}$ \\
\hline
\end{tabular}

Note. - This table follows the contents Table 1 in Paper IV. We denote the samples monitored during the 2012-2013, 2013-2014 and 2014-2015 observing seasons as SEAMBH2012, SEAMBH2013 and SEAMBH2014, respectively. $N_{\text {spec }}$ is the numbers of spectroscopic epochs, $R_{*}$ is the angular distance between the object and the comparison star and PA the position angle from the AGN to the comparison star. We marked the time lag of J080131 as "uncertain" in PaperIV, however we pick it up here because its lag reported in Paper IV is highly consistent with the number measured in the present paper.

of correlation as the normal $R_{\mathrm{H} \beta}-L_{5100}$ relationship. The constants $\alpha_{0}$ and $\beta_{0}$ differ slightly from one study to the next, depending on the number of sources and their exact luminosity range (e.g., Kilerci Eser et al. 2015). For sub-Eddington accreting AGNs, $\alpha_{0}=35.5 \mathrm{ltd}$ and $\beta_{0}=0.53$, but for SEAMBHs they are different (see Paper IV).

As reported in Paper IV, some objects from the SEAMBH2012 and SEAMBH2013 samples have much shorter $\mathrm{H} \beta$ lags compared with objects with similar luminosity, and the $R_{\mathrm{H} \beta}-L_{5100}$ relation has a large scatter if they are included. In particular, the reduction of the lags increases with the dimensionless accretion rate, defined as $\dot{\mathscr{M}}=\dot{M}_{\bullet} / L_{\text {Edd }} c^{-2}$, where $\dot{M}_{\bullet}$ is the accretion rate, $L_{\text {Edd }}$ is the Eddington luminosity and $c$ is the speed of light. Furthermore, it has been found, so far in the present campaigns, that SEAMBHs have a range of accretion rates from a few to $\sim 10^{3}$. This kind of shortened $\mathrm{H} \beta$ lags was discovered in the current SEAMBH project (a comparison with previous campaigns is given in Section 6.5). Such high accretion rates are characteristic of the regime of slim accretion disks (Abramowicz et al. 1988; Szuszkiewicz et al. 1996; Wang \& Zhou 1999; Wang et al. 1999; Mineshige et al. 2000; Wang \& Netzer 2003; Sadowski 2009). These interesting properties needed to be confirmed with observations. We aim to explore whether we can define a new scaling relation, $R_{\mathrm{H} \beta}=R_{\mathrm{H} \beta}\left(L_{5100}, \dot{\mathscr{M}}\right)$, which links the size of the BLR to both the AGN luminosity and accretion rate.

We report new results from SEAMBH2014. We describe target selection, observation details and data reduction in $\$ 2$. $\mathrm{H} \beta$ lags, $\mathrm{BH}$ mass and accretion rates are provided in $\S 3$. Properties of $\mathrm{H} \beta$ lags are discussed in $\$ 4$, and a new scaling relation of $\mathrm{H} \beta$ lags is established in $\$ 5$. Section 6 introduces the fundamental plane, which is used to estimate accretion rates from single-epoch spectra, for application of the new size-luminosity scaling relation of the BLR. Brief discus- sions of the shortened lags are presented in $\$ 7$. We draw conclusions in $\S 8$. Throughout this work we assume a standard $\Lambda \mathrm{CDM}$ cosmology with $H_{0}=67 \mathrm{~km} \mathrm{~s}^{-1} \mathrm{Mpc}^{-1}, \Omega_{\Lambda}=0.68$ and $\Omega_{M}=0.32$ (Ade et al. 2014).

\section{OBSERVATIONS AND DATA REDUCTION}

\subsection{Target Selection}

We followed the procedures for selecting SEAMBH candidates described in Paper IV. We used the fitting procedures to measure $\mathrm{H} \beta$ profile and $5100 \AA$ luminosity of SDSS quasar spectra described by Hu et al. (2008a,b). Following the standard assumption that the BLR gas is virialized, we estimate the $\mathrm{BH}$ mass as

$$
M_{\bullet}=f_{\mathrm{BLR}} \frac{R_{\mathrm{H} \beta} V_{\mathrm{FWHM}}^{2}}{G}=1.95 \times 10^{6} f_{\mathrm{BLR}} V_{3}^{2} \tau_{10} M_{\odot},
$$

where $R_{\mathrm{H} \beta}=c \tau_{\mathrm{H} \beta}, \tau_{\mathrm{H} \beta}$ is the $\mathrm{H} \beta$ lag measured in the rest frame, $\tau_{10}=\tau_{\mathrm{H} \beta} / 10$ days, $G$ is the gravitational constant, and $V_{3}=V_{\mathrm{FWHM}} / 10^{3} \mathrm{~km} \mathrm{~s}^{-1}$ is the full-width-half-maximum (FWHM) of the $\mathrm{H} \beta$ line profile in units of $10^{3} \mathrm{~km} \mathrm{~s}^{-1}$. We take the virial factor $f_{\text {BLR }}=1$ in our series of papers (see some discussions in Paper IV).

In order to select AGNs with high accretion rates, we employed the formulation of accretion rates derived from the standard disk model of Shakura \& Sunyaev (1973). In the standard model it is assumed that the disk gas is rotating with Keplerian angular momentum, and thermal equilibrium is localized between viscous dissipation and blackbody cooling. Observationally, this model is supported from fits of the socalled big blue bump in quasars (Czerny \& Elvis 1987; Wandel \& Petrosian 1988; Sun \& Malkan 1989; Laor \& Netzer 1989; Collin et al. 2002; Brocksopp et al. 2006; Kishimoto et al. 2008; Davis \& Laor 2011; Capellupo et al. 2015). The 
Table 2

Light curves of J075949 and J080131

\begin{tabular}{|c|c|c|c|c|c|c|c|c|c|}
\hline \multicolumn{5}{|c|}{ J075949 } & \multicolumn{5}{|c|}{ J080131 } \\
\hline \multicolumn{2}{|c|}{ Photometry } & \multicolumn{3}{|c|}{ Spectra } & \multicolumn{2}{|c|}{ Photometry } & \multicolumn{3}{|c|}{ Spectra } \\
\hline JD & mag & JD & $F_{5100}$ & $F_{\mathrm{H} \beta}$ & JD & mag & JD & $F_{5100}$ & $F_{\mathrm{H} \beta}$ \\
\hline 29.374 & $17.373 \pm 0.007$ & 76.365 & $2.773 \pm 0.029$ & $2.469 \pm 0.038$ & 60.324 & $17.757 \pm 0.010$ & 112.294 & $2.096 \pm 0.016$ & $0.853 \pm 0.027$ \\
\hline 30.360 & $17.375 \pm 0.008$ & 80.319 & $2.864 \pm 0.017$ & $2.461 \pm 0.031$ & 62.314 & $17.720 \pm 0.010$ & 116.394 & $2.076 \pm 0.016$ & $0.893 \pm 0.029$ \\
\hline 32.352 & $17.406 \pm 0.009$ & 83.314 & $2.724 \pm 0.038$ & $2.366 \pm 0.041$ & 63.299 & $17.734 \pm 0.010$ & 119.336 & $2.106 \pm 0.021$ & $0.831 \pm 0.031$ \\
\hline 33.339 & $17.416 \pm 0.009$ & 86.422 & $2.795 \pm 0.011$ & $2.535 \pm 0.023$ & 68.397 & $17.721 \pm 0.013$ & 135.324 & $2.086 \pm 0.026$ & $0.807 \pm 0.033$ \\
\hline 34.331 & $17.408 \pm 0.009$ & 89.378 & $2.888 \pm 0.012$ & $2.402 \pm 0.032$ & 77.318 & $17.746 \pm 0.009$ & 139.319 & $2.074 \pm 0.028$ & $0.862 \pm 0.035$ \\
\hline
\end{tabular}

Note. - JD: Julian dates from 2,456,900; $F_{5100}$ and $F_{\mathrm{H} \beta}$ are fluxes at $(1+z) 5100 \AA$ and $\mathrm{H} \beta$ emission lines in units of $10^{-16} \mathrm{erg} \mathrm{s}^{-1} \mathrm{~cm}^{-2}{ }^{-1}$ and $10^{-14} \mathrm{erg} \mathrm{s}^{-1} \mathrm{~cm}^{-2}$. (This table is available in its entirety in a machine-readable form in the online journal. A portion is shown here for guidance regarding its form and content.)

dimensionless accretion rate is given by

$$
\dot{\mathscr{M}}=20.1\left(\frac{\ell_{44}}{\cos i}\right)^{3 / 2} m_{7}^{-2} \text {, }
$$

where $m_{7}=M_{\bullet} / 10^{7} M_{\odot}$ (see Papers II and IV) and $i$ is the inclination angle to the line of sight of the disk. We take $\cos i=0.75$, which represents a mean disk inclination for a type 1 AGNs with a torus covering factor of about 0.5 (it is assumed that the torus axis is co-aligned with the disk axis). Previous studies estimate $i \approx 0-45^{\circ}$ [e.g., Fischer et al. (2014) find a inclination range of $i \approx 10^{\circ}-45^{\circ}$, whereas Pancoast et al. (2014) quote $i \approx 5^{\circ}-45^{\circ}$; see also supplementary materials in Shen \& Ho (2014)], which results in $\Delta \log \dot{\mathscr{M}}=1.5 \Delta \log \cos i \lesssim 0.15$ from Equation (3). This uncertainty is significantly smaller than the average uncertainty on $\dot{\mathscr{M}}(\sim 0.3-0.5 \mathrm{dex})$ in the present paper, and is thus ignored. Equation (3) applies to AGNs that have accretion rates $10^{-2} \lesssim \dot{\mathscr{M}} \lesssim 3 \times 10^{3}$, namely excluding the regimes of advection-dominated accretion flows (ADAF; Narayan \& Yi 1994) and of flows with hyperaccretion rates $\left(\dot{\mathscr{M}} \geq 3 \times 10^{3}\right.$; see Appendix A for the validity of Equation 3 for SEAMBHs).

Using the normal $R_{\mathrm{H} \beta}-L_{5100}$ relation (Bentz et al. 2013), we fitted all the quasar spectra in SDSS Data Release 7 by the procedures in $\mathrm{Hu}$ et al. (2008a, b) and applied Equations (2) and (3) to select high- $\dot{\mathscr{M}}$ targets. We ranked quasars in terms of $\dot{\mathscr{M}}$ and chose ones as candidates with the highest $\dot{\mathscr{M}}$. We found that the high- $\dot{\mathscr{M}}$ quasars are characterized by 1) strong optical $\mathrm{Fe}$ II lines; 2) relatively narrow $\mathrm{H} \beta$ lines $\left(\lesssim 2000 \mathrm{~km} \mathrm{~s}^{-1}\right)$; 3) weak [O III] lines; and 4) steep 2-10 keV spectra (Wang et al. 2004). These properties are similar to those of NLS1s (Osterbrock \& Pogge 1987; Boroson \& Green 1992), but most of the candidates have more extreme accretion rates (a detailed comparison of SEAMBH properties with normal quasars will be carried out in a separate paper). Considering that the lags of all targets should be measured within one observing season, and taking into consideration the limitations of the weather of the Lijiang Station of Yunnan Observatory (periods between June and September are raining seasons there), we only chose objects with maximum estimated lags of about 100 days or so (the monitoring periods should be at least a few times the presumed lags). Also, to ensure adequate signal-to-noise ratio $(\mathrm{S} / \mathrm{N})$ for measurements of light curves, we restricted the targets to a redshift range of $z=0.1-0.3$ and magnitudes $r^{\prime} \leq 18.0$. The fraction of radio-loud objects with $\dot{\mathscr{M}}>3$ is not high. We discarded radio-loud objectsT0 based on available FIRST observations, in order to avoid $\mathrm{H} \beta$ reverberations potentially affected by nonthermal emission from relativistic jets, or optical continuum emission strongly contaminated by jets. We chose about 20 targets for photometry monitoring, which served as a preselection to trigger follow-up spectroscopic monitoring. The photometric monitoring yielded 10 targets with significant variations ( $\gtrsim 0.1$ magnitudes), and time lags were successfully measured for 5 objects (Table 1). For an overview of our entire ongoing campaign, Table 1 also lists samples from SEAMBH2012 and SEAMBH2013.

To summarize: we have selected about 30 targets for spectroscopic monitoring during the last three years (2012-2014). The successful rate of the monitoring project is about $2 / 3$. Our failure to detect a lag for the remaining $1 / 3$ of the sample are either due to low-amplitude variability or bad weather that leads to poor monitoring cadence. In particular, the SEAMBH2014 observations were seriously affected by the El Niño phenomenon.

\subsection{Photometry and Spectroscopy}

The SEAMBH project uses the Lijiang $2.4 \mathrm{~m}$ telescope, which has an alt-azimuth Ritchey-Chrétien mount with a field de-rotator that enables two objects to be positioned along the same long slit. It is located in Lijiang and is operated by Yunnan Observatories. We adopted the same observational procedures described in detail in Paper I, which also introduces the telescope and spectrograph. We employed the Yunnan Faint Object Spectrograph and Camera (YFOSC), which has a back-illuminated $2048 \times 4608$ pixel CCD covering a field of $10^{\prime} \times 10^{\prime}$. During the spectroscopic observation, we put the target and a nearby comparison star into the slit simultaneously, which can provide high-precision flux calibration. As in SEAMBH2013 (Paper IV), we adopted a 5"-wide slit to minimize the influence of atmospheric differential refraction, and used Grism 3 with a spectral resolution of $2.9 \AA /$ pixel and wavelength coverage of 3800-9000 A. To check the accuracy of spectroscopic calibration, we performed differential photometry of the targets using some other stars in the same field. We used an SDSS $r^{\prime}$-band filter for photometry to avoid the potential contamination by emission lines such as $\mathrm{H} \beta$ and $\mathrm{H} \alpha$. Photometric and spectroscopic exposure times are typically 10 and 60 min, respectively.

10 It has been realised that high-accretion rate AGNs are usually radioquiet (Greene \& Ho 2006), although there are a few NLS1s reported to be radio-loud. The fraction of radio-loud AGNs decreases with increasing accretion rate (Ho 2002, 2008). 
Table 3

Light curves of J084533 and J085946

\begin{tabular}{|c|c|c|c|c|c|c|c|c|c|}
\hline \multicolumn{5}{|c|}{ J084533 } & \multicolumn{5}{|c|}{ J085946 } \\
\hline \multicolumn{2}{|c|}{ Photometry } & \multicolumn{3}{|c|}{ Spectra } & \multicolumn{2}{|c|}{ Photometry } & \multicolumn{3}{|c|}{ Spectra } \\
\hline JD & mag & $\mathrm{JD}$ & $F_{5100}$ & $F_{\mathrm{H} \beta}$ & JD & mag & JD & $F_{5100}$ & $F_{\mathrm{H} \beta}$ \\
\hline 29.417 & $17.803 \pm 0.008$ & 75.402 & $1.728 \pm 0.026$ & $1.145 \pm 0.047$ & 30.428 & $17.401 \pm 0.008$ & 90.382 & $2.651 \pm 0.015$ & $1.693 \pm 0.024$ \\
\hline 30.398 & $17.797 \pm 0.008$ & 80.395 & $1.721 \pm 0.019$ & $1.085 \pm 0.033$ & 33.403 & $17.399 \pm 0.007$ & 97.433 & $2.593 \pm 0.033$ & $1.728 \pm 0.038$ \\
\hline 33.376 & $17.777 \pm 0.009$ & 84.365 & $1.680 \pm 0.020$ & $1.046 \pm 0.035$ & 36.410 & $17.406 \pm 0.005$ & 104.293 & $2.392 \pm 0.024$ & $1.835 \pm 0.034$ \\
\hline 36.368 & $17.780 \pm 0.009$ & 91.326 & $1.673 \pm 0.016$ & $1.086 \pm 0.028$ & 48.357 & $17.422 \pm 0.008$ & 111.261 & $2.583 \pm 0.022$ & $1.595 \pm 0.037$ \\
\hline 38.411 & $17.816 \pm 0.017$ & 104.409 & $1.681 \pm 0.015$ & $1.069 \pm 0.029$ & 51.353 & $17.440 \pm 0.006$ & 116.448 & $2.512 \pm 0.025$ & $1.697 \pm 0.027$ \\
\hline
\end{tabular}

Note. - This table is available in its entirety in a machine-readable form in the online journal. A portion is shown here for guidance regarding its form and
content.

The reduction of the photometry data was done in a standard way using IRAF routines. Photometric light curves were produced by comparing the instrumental magnitudes to those of standard stars in the field (see, e.g., Netzer et al. 1996, for details). The radius for the aperture photometry is typically $\sim 4^{\prime \prime}$ (seeing $\left.\sim 1.5^{\prime \prime}-2^{\prime \prime}\right)$, and background is determined from an annulus with radius $8^{\prime \prime} .5$ to $17^{\prime \prime}$. The uncertainties on the photometric measurements include the fluctuations due to photon statistics and the scatter in the measurement of the stars used.

The spectroscopic data were also reduced with IRAF. The extraction width is fixed to $8^{\prime \prime} .5$, and the sky regions are set to $7^{\prime \prime} .4-14^{\prime \prime} .1$ on both sides of the extracted region. The average $\mathrm{S} / \mathrm{N}$ of the $5100 \AA$ continuum of individual spectra are from $\sim 16$ to $\sim 22$, except for J085946, which only has S/N $\approx 12$. The flux of spectroscopic data was calibrated by simultaneously observing a nearby comparsion star along the slit (see Paper I). The fiducial spectra of the comparison stars are generated using observations from several nights with the best weather conditions. The absolute fluxes of the fiducial spectra are calibrated using additional spectrophotometric standard stars observed in those nights. Then, the in-slit comparison stars are used as standards to calibrate the spectra of targets observed in each night. The sensitivity as a function of wavelength is produced by comparing the observed spectrum of the comparison star to its fiducial spectrum. Finally, the sensitivity function is applied to calibrate the observed AGN spectrum 11 . The procedures adopted here resemble the method used by Maoz et al. (1990) and Kaspi et al. (2000). In order to illustrate the invariance of the comparison stars, we show the light curves from the differential photometry of the comparison stars in Appendix B. It is clear that their fluxes are very stable and the variations are less than $\sim 1 \%$.

The calibration method of van Groningen \& Wanders (1992), based on the [O III] emission line and popularily used in many RM campaigns (e.g., Peterson et al. 1998; Bentz et al. 2009; Denney et al. 2010; Grier et al. 2012; Barth et al. 2015), is not suitable for SEAMBHs. [O III] $\lambda 5007$ tends to be weak in SEAMBHs (especially for the objects in SEAMBH2013-2014), and, even worse, is blended with strong Fe II around $5016 \AA$. Applying the calibration method to SEAMBHs results in large statistical (caused by the weakness of [O III]) and systematic (caused by the variability of Fe II; see Paper III) uncertainties. The method based on inslit comparison stars, used in our campaign, does not rely on

\footnotetext{
11 The uncertainty of our absolute flux calibration is $\lesssim 10 \%$. We multiply the fiducial spectra of the in-slit stars with the bandpass of the SDSS $\mathrm{r}^{\prime}$ filter and compare their synthesized magnitudes with the magnitudes found in the SDSS database. The maximum difference is $\lesssim 10 \%$.
}

[O III] and provides accurate flux calibration for the spectra of SEAMBHs. For comparison, in Paper I we measured the [O III] fluxes in the calibrated spectra of three objects in SEAMBH2012 with relatively strong [O III]; the variation of their [O III] flux is on the order of $\sim 3 \%$. This clearly demonstrates the robustness of our flux calibration method based on in-slit comparison stars.

The procedures to measure the $5100 \AA$ and $\mathrm{H} \beta$ flux are nearly the same as those given in Paper I. The continuum beneath $\mathrm{H} \beta$ line is determined by interpolation of two nearby bands (4740-4790 $\AA$ and $5075-5125 \AA$ ) in the rest frame. These two bands have minimal contamination from emission lines. The flux of $\mathrm{H} \beta$ is measured by integrating the band between 4810 and $4910 \AA$ after subtraction of the continuum; the $\mathrm{H} \beta$ band is chosen to avoid the influence from Fe II lines. The $5100 \AA$ flux is taken to be the median over the region 5075-5125 $\AA$. Detailed information of the observations is provided in Table 1 . All the photometry and continuum and $\mathrm{H} \beta$ light curves for the five objects with successfully detected lags are listed in Tables 2-4 and shown in Figure 1. We also calculated the mean and RMS (root mean square) spectra and present them in Appendix C.

\subsection{Host Galaxies}

Like the SEAMBH2013 sample, we have no observations that can clearly separate the host galaxies of the AGNs in SEAMBH2014. Shen et al. (2011) propose the following empirical relation to estimate the fractional contribution of the host galaxy to the optical continuum emission: $L_{5100}^{\text {host }} / L_{5100}^{\mathrm{AGN}}=$ $0.8052-1.5502 x+0.912 x^{2}-0.1577 x^{3}$, for $x<1.053$, where $x=\log \left(L_{5100}^{\mathrm{tot}} / 10^{44} \mathrm{erg} \mathrm{s}^{-1}\right)$ and $L_{5100}^{\mathrm{tot}}$ is the total emission from the AGN and its host at $5100 \AA$. For $x>1.053, L_{5100}^{\text {host }} \ll$ $L_{5100}^{\mathrm{AGN}}$, and the host contamination can be neglected. The host fractions at $5100 \AA$ for the objects (J075949, J080131, $\mathrm{J} 084533$, J085946 and J102339) are (27.5\%, 37.1\%, 14.2\%, $19.0 \%$ and $31.8 \%$ ). The values of $L_{5100}$ listed in Table 5 are the host-subtracted luminosities. We note that this empirical relation is based on SDSS spectroscopic observations with a $3^{\prime \prime}$ fiber, whereas we used a $5^{\prime \prime}$-wide slit. It should apply to our observations reasonably well (see Paper IV for additional discussions on this issue). We will revisit this issue in the future using high-resolution images that can more reliably separate the host.

\section{MEASUREMENTS OF H $\beta$ LAGS, BLACK HOLE MASSES AND} ACCRETION RATES

\subsection{Lags}



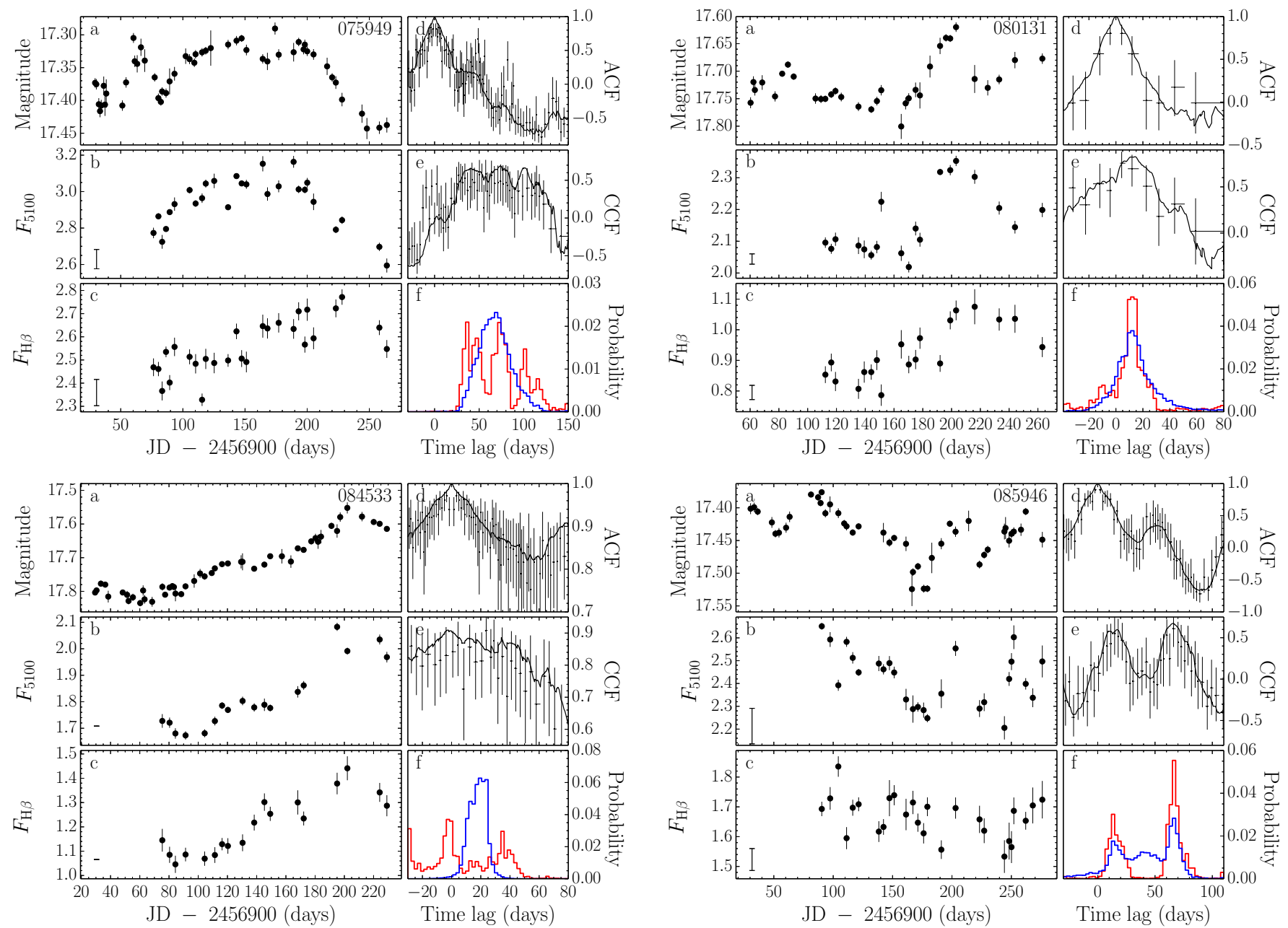

Figure 1. Light curves and cross-correlation results. Each object has six panels: $(a, b, c)$ are light curves of SDSS $r^{\prime}$-band magnitude, $5100 \AA$ continuum and $\mathrm{H} \beta$ emission, respectively; $(d, e, f)$ are auto-correlation function (ACF) of the $r^{\prime}$-band magnitude (5100 ̊̊ continuum for J080131; see the main text), cross-correlation function (CCF) of the $r^{\prime}$-band magnitude and $\mathrm{H} \beta$ line emission (5100 $\AA$ and $\mathrm{H} \beta$ for J080131) and the Monte Carlo simulations of peak (red) and centroid (blue) of the lags, respectively. In panels $d$ and $e$, the solid lines show the results of the ICCF method, and the points with error bars are from ZDCF (Z-transformed discrete correlation function). $F_{5100}$ and $F_{\mathrm{H} \beta}$ are in units of $10^{-16} \mathrm{erg} \mathrm{s}^{-1} \mathrm{~cm}^{-2-1}$ and $10^{-14} \mathrm{erg} \mathrm{s}^{-1} \mathrm{~cm}^{-2}$, respectively. Bars with terminals denote systematic errors and are plotted in the corners of the panels (see Paper I for details). For J084533, the systematic error bars are so small that the caps of error bars merge in panels $b$ and $c$; the same holds for $\mathrm{J} 102339$ in panel $b$.

As in Papers I-IV, we used cross-correlation analysis to determine $\mathrm{H} \beta$ lags relative to photometric or $5100 \AA$ continuum light curves. We use the centroid lag for $\mathrm{H} \beta$. The uncertainties on the lags are determined through the "flux randomization/random subset sampling" method (RS/RSS; Peterson et al. 1998, 2004). The cross-correlation centroid distribution (CCCD, described in Appendix E) and cross-correlation peak distribution (CCPD) generated by the FR/RSS method (Maoz \& Netzer 1989; Peterson et al. 1998, 2004; Denney et al. 2006, 2010, and references therein) are shown in Figure 1. We used the following criteria to define a successful detection of $\mathrm{H} \beta$ lag: 1) non-zero lag from the CCF peak and 2) a maximum correlation coefficient larger than 0.5. Data for the light curves of the targets are given in Tables 2-4. All the measurements of the SEAMBH2014 sample are provided in Table 5.

The $r^{\prime}$-band light curves are generally consistent with the $5100 \AA$ A continuum light curve, but the former usually have small scatter, as shown in Figure 1. We calculated CCFs for the $\mathrm{H} \beta$ light curves with both $r^{\prime}$-band photometry and with $5100 \AA$ spectral continuum for all objects. The quality of the
$\mathrm{H} \beta-r^{\prime}$ CCFs is usually better than the $\mathrm{H} \beta-F_{5100}$ CCFs. We show the $\mathrm{H} \beta-r^{\prime} \mathrm{CCFs}$ for all objects in Figure 1, except for J080131. We use the $\mathrm{H} \beta-r^{\prime}$ lags in the following analysis. For J080131, the $r^{\prime}$-band light curve between 200 and 220 days does not match the $5100 \AA$ continuum light curve, even though $\mathrm{H} \beta$ does follow $5100 \AA$ continuum tightly. Notes to individual sources are given in Appendix D.

\subsection{Black Hole Masses and Accretion Rates}

There are two ways of calculating BH mass, base either on the RMS spectrum (e.g., Peterson et al. 2004; Bentz et al. 2009; Denney et al. 2010; Grier et al. 2012) or on the mean spectrum (e.g., Kaspi et al. 2005; Papers I-IV). Different studies also adopt different measures of the line width, typically either the line dispersion $\sigma_{\text {line }}$ (second moment of the line profile) or the FWHM. In this study, we choose to parameterize the line width using FWHM, as measured in the mean spectra. The narrow $\mathrm{H} \beta$ component may influence the measurement of FWHM. We adopt the same procedure as in Paper I to remove the narrow $\mathrm{H} \beta$. We fix narrow $\mathrm{H} \beta /[\mathrm{O}$ III] $\lambda 5007$ to 0.1 , and measure FWHM from the mean spectra with narrow $\mathrm{H} \beta$ subtracted. Then we set $\mathrm{H} \beta /[\mathrm{O}$ III $] \lambda 5007$ to 0 and 0.2 and 


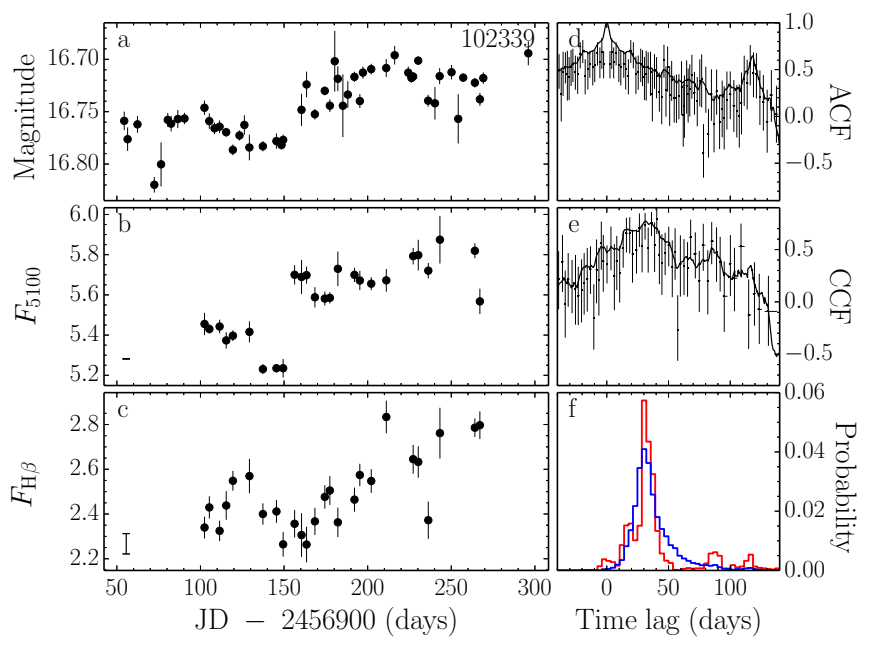

Figure 1 continued.

repeat the process to obtain lower and upper limits to FWHM. The relatively wide slit employed in our campaign $\left(5^{\prime \prime}\right)$ significantly broadens the emission lines by $V_{\text {inst }} \approx 1200 \mathrm{~km} \mathrm{~s}^{-1}$, where $V_{\text {inst }}$ is the instrumental broadening that can be estimated from the broadening of selected comparison stars. As in Paper IV, we obtain the intrinsic width of the mean spectra from FWHM $=\left(\mathrm{FWHM}_{\mathrm{obs}}^{2}-V_{\text {inst }}^{2}\right)^{1 / 2}$. The FWHM simply obtained here is accurate enough for $\mathrm{BH}$ mass estimation. Our procedure for $\mathrm{BH}$ mass estimation is based on FWHM measured from the mean spectrum (see explanation in Papers I and II). As shown recently in Woo et al. (2015), the scatter in the scaling parameter $\left(f_{\mathrm{BLR}}\right)$ derived in this method is very similar to the scatter in the method based on the RMS spectrum. We use Equations (2) and (3) to calculate accretion rates and $\mathrm{BH}$ masses for the five sources listed in Table 5. For convenience and completeness, Table 5 also lists $\mathrm{H} \beta$ lags, $\mathrm{BH}$ masses and accretion rates for the sources from SEAMBH2012 and SEAMBH2013. Our campaign has successfully detected $\mathrm{H} \beta$ lags for 18 SEAMBHs since October 2012.

As described in Paper II, there are some theoretical uncertainties in identifying a critical value of $\dot{\mathscr{M}}$ to define a SEAMBH (Laor \& Netzer 1989; Beloborodov 1998; Sadowski et al. 2011). Following Paper II, we classified SEAMBHs as those objects with $\eta \mathscr{\mathscr { M }} \geq 0.1$. This is based on the idea that beyond this value, the accretion disk becomes slim and the radiation efficiency is reduced mainly due to photon trapping (Sadowski et al. 2011). Since we currently cannot observe the entire spectral energy distribution, we have no direct way to measure $L_{\mathrm{bol}} / L_{\mathrm{Edd}}$, and this criterion is used as an approximate tool to identify SEAMBH candidates. To be on the conservative side, we chose the lowest possible efficiency, $\eta=0.038$ (retrograde disk with $a=-1$; see Bardeen et al. 1972). Thus, SEAMBHs are objects with $\dot{\mathscr{M}}=2.63$. For simplicity, in this paper we use $\dot{\mathscr{M}}_{\text {min }}=3$ as the required minimum (Papers II and IV). We refer to AGNs with $\dot{\mathscr{M}} \geq 3$ as SEAMBHs and those with $\dot{\mathscr{M}}<3$ as sub-Eddington ones. Paper IV clearly shows that the properties of the $R_{\mathrm{H} \beta}-L_{5100}$ relation for $\dot{\mathscr{M}} \geq 3$ and $\dot{\mathscr{M}}<3$ are significantly different.

Figure 2 plots distributions of $L_{5100}, \operatorname{EW}(\mathrm{H} \beta), \dot{\mathscr{M}}$ and $M_{\bullet}$ of all the mapped AGNs (41 from Bentz et al. 2013 and the 18
Table 4

Light curves of J102339

\begin{tabular}{|c|c|c|c|c|}
\hline \multicolumn{5}{|c|}{ J102339 } \\
\hline \multicolumn{2}{|c|}{ Photometry } & \multicolumn{3}{|c|}{ Spectra } \\
\hline JD & mag & JD & $F_{5100}$ & $F_{\mathrm{H} \beta}$ \\
\hline 54.400 & $16.759 \pm 0.009$ & 102.431 & $5.455 \pm 0.056$ & $2.340 \pm 0.049$ \\
\hline 56.431 & $16.776 \pm 0.011$ & 105.309 & $5.430 \pm 0.022$ & $2.429 \pm 0.050$ \\
\hline 62.333 & $16.762 \pm 0.008$ & 111.450 & $5.443 \pm 0.034$ & $2.325 \pm 0.046$ \\
\hline 72.412 & $16.820 \pm 0.007$ & 115.406 & $5.374 \pm 0.041$ & $2.438 \pm 0.064$ \\
\hline 76.438 & $16.800 \pm 0.021$ & 119.396 & $5.397 \pm 0.027$ & $2.548 \pm 0.044$ \\
\hline
\end{tabular}

Note. - This table is available in its entirety in a machine-readable form in the online journal. A portion is shown here for guidance regarding its form and content.

SEAMBHs from our campaign; see Table 7 in Paper IV and Table 5 here). As shown clearly in the diagrams, SEAMBH targets are generally more luminous by a factor of 2-3 compared to previous RM AGNs (Figure $2 a$ ). The BH masses of SEAMBHs are generally less smaller by a factor of $10 \mathrm{com}-$ pared to previous samples, whereas, as a consequence of our selection, the accretion rates of SEAMBHs are higher by 2-3 orders of magnitude (Figures $2 c$ and $2 d$ ). However, $\mathrm{EW}(\mathrm{H} \beta)$ of SEAMBHs are not significantly smaller (Figure $2 d$ ). On average, the high- $\dot{\mathscr{M}}$ sources have lower mean $\operatorname{EW}(\mathrm{H} \beta)$, consistent with the inverse correlation between $\operatorname{EW}(\mathrm{H} \beta)$ and $L_{\text {bol }} / L_{\text {Edd }}$ (e.g., Netzer et al. 2004).

\section{PROPERTIES OF H $\beta$ LAGS IN SEAMBHS}

The $R_{\mathrm{H} \beta}-L_{5100}$ correlation was originally presented by Peterson (1993; his Figure 10, only nine objects). It was confirmed by Kaspi et al. (2000) using a sample of 17 lowredshift quasars. Bentz et al. (2013) refined the $R_{\mathrm{H} \beta}-L_{5100}$ relation through subtraction of host contamination and found that its intrinsic scatter is only 0.13 dex. Paper IV (Table 7) provides a complete list of previously mapped AGNs, based on Bentz et al. (2013); we directly use these values. As in Paper IV, for objects with multiple measurements of $\mathrm{H} \beta$ lags, we obtain the $\mathrm{BH}$ mass from each campaign and then calculate the average $\mathrm{BH}$ mass. Using the averaged $\mathrm{BH}$ mass, we apply it to get accretion rates of the BHs during each monitoring epoch, which are further averaged to obtain the mean accretion rates of those objects (Kaspi et al. 2005; Bentz et al. 2013). We call this the "average scheme." On the other hand, we may consider each individual measurement of a single object as different objects (e.g., Bentz et al. 2013). We called this the "direct scheme." Although the two approaches are in principle different, we obtain very similar results (see a comparison in Paper IV).

All correlations of two parameters shown in this paper are calculated with the FITEXY method, using the version adopted by Tremaine et al. (2002), which allows for intrinsic scatter by increasing the uncertainties in small steps until $\chi^{2}$ reaches unity (this is typical for many of our correlations). We also emplot the BCES method (Akristas \& Bershady 1996) but prefer not to use its results because it is known to give unreliable results in samples containing outliers (there are a few objects with quite large uncertainties of $\dot{\mathscr{M}})$.

$$
\text { 4.1. The } R_{\mathrm{H} \beta}-L_{5100} \text { relation }
$$

12 NGC 7469 was mapped twice by Collier et al. (1998) and Peterson et al. (2014). While their $\mathrm{H} \beta$ lags are consistent, the FWHM of $\mathrm{H} \beta$ is very different. We only retain the later observation in the analysis. 
Table 5

$\mathrm{H} \beta$ Reverberations of the SEAMBHs

\begin{tabular}{|c|c|c|c|c|c|c|c|c|}
\hline \multirow[t]{2}{*}{ Objects } & $\tau_{\mathrm{H} \beta}$ & FWHM & $\sigma_{\text {line }}$ & $\log \left(M_{\bullet} / M_{\odot}\right)$ & $\log \ddot{\mathscr{M}}$ & $\log L_{5100}$ & $\log L_{\mathrm{H} \beta}$ & $\mathrm{EW}(\mathrm{H} \beta)$ \\
\hline & (days) & $\left(\mathrm{km} \mathrm{s}^{-1}\right)$ & $\left(\mathrm{km} \mathrm{s}^{-1}\right)$ & & & $\left(\mathrm{erg} \mathrm{s}^{-1}\right)$ & $\left(\operatorname{erg~s}^{-1}\right)$ & $(\AA)$ \\
\hline \multicolumn{9}{|c|}{ SEAMBH2012 } \\
\hline Mrk 335 & $8.7_{-1.9}^{+1.6}$ & $2096 \pm 170$ & $1470 \pm 50$ & $6.87_{-0.14}^{+0.10}$ & $1.28_{-0.30}^{+0.37}$ & $43.69 \pm 0.06$ & $42.03 \pm 0.06$ & $110.5 \pm 22.3$ \\
\hline Mrk 1044 & $10.5_{-2.7}^{+3.3}$ & $1178 \pm 22$ & $766 \pm 8$ & $6.45_{-0.13}^{+0.12}$ & $1.22_{-0.41}^{+0.40}$ & $43.10 \pm 0.10$ & $41.39 \pm 0.09$ & $101.4 \pm 31.9$ \\
\hline Mrk 382 & $7.5_{-2.0}^{+2.9}$ & $1462 \pm 296$ & $840 \pm 37$ & $6.50_{-0.29}^{+0.19}$ & $1.18_{-0.53}^{+0.69}$ & $43.12 \pm 0.08$ & $41.01 \pm 0.05$ & $39.6 \pm 9.0$ \\
\hline Mrk 142 & $7.9_{-1.1}^{+1.2}$ & $1588 \pm 58$ & $948 \pm 12$ & $6.59_{-0.07}^{+0.07}$ & $1.65_{-0.23}^{+0.23}$ & $43.56 \pm 0.06$ & $41.60 \pm 0.04$ & $55.2 \pm 9.5$ \\
\hline IRAS F12397 & $9.7_{-1.8}^{+5.5}$ & $1802 \pm 560$ & $1150 \pm 122$ & $6.79_{-0.45}^{+0.27}$ & $2.26_{-0.62}^{+0.98}$ & $44.23 \pm 0.05$ & $42.26 \pm 0.04$ & $54.2 \pm 8.4$ \\
\hline Mrk 486 & $23.7_{-2.7}^{+7.5}$ & $1942 \pm 67$ & $1296 \pm 23$ & $7.24_{-0.06}^{+0.12}$ & $0.55_{-0.32}^{+0.20}$ & $43.69 \pm 0.05$ & $42.12 \pm 0.04$ & $135.9 \pm 20.3$ \\
\hline Mrk 493 & $11.6_{-2.6}^{+1.2}$ & $778 \pm 12$ & $513 \pm 5$ & $6.14_{-0.11}^{+0.04}$ & $1.88_{-0.21}^{+0.33}$ & $43.11 \pm 0.08$ & $41.35 \pm 0.05$ & $87.4 \pm 18.1$ \\
\hline IRAS 04416 & $13.3_{-1.4}^{+13.9}$ & $1522 \pm 44$ & $1056 \pm 29$ & $6.78_{-0.06}^{+0.31}$ & $2.63_{-0.67}^{+0.16}$ & $44.47 \pm 0.03$ & $42.51 \pm 0.02$ & $55.8 \pm 4.7$ \\
\hline \multicolumn{9}{|c|}{ SEAMBH2013 } \\
\hline SDSS J075101 & $33.4_{-5.6}^{+15.6}$ & $1495 \pm 67$ & $1055 \pm 32$ & $7.16_{-0.09}^{+0.17}$ & $1.34_{-0.41}^{+0.25}$ & $44.12 \pm 0.05$ & $42.25 \pm 0.03$ & $68.1 \pm 8.6$ \\
\hline SDSS J080101 & $8.3_{-2.7}^{+9.7}$ & $1930 \pm 18$ & $1119 \pm 3$ & $6.78_{-0.17}^{+0.34}$ & $2.33_{-0.72}^{+0.39}$ & $44.27 \pm 0.03$ & $42.58 \pm 0.02$ & $105.5 \pm 8.3$ \\
\hline SDSS J080131 & $11.5_{-3.6}^{+8.4}$ & $1188 \pm 3$ & $850 \pm 12$ & $6.50_{-0.16}^{+0.24}$ & $2.46_{-0.54}^{+0.38}$ & $43.98 \pm 0.04$ & $42.08 \pm 0.03$ & $64.0 \pm 7.0$ \\
\hline SDSS J081441 & $18.4_{-8.4}^{+12.7}$ & $1615 \pm 22$ & $1122 \pm 11$ & $6.97_{-0.27}^{+0.23}$ & $1.56_{-0.57}^{+0.63}$ & $44.01 \pm 0.07$ & $42.42 \pm 0.03$ & $132.0 \pm 23.7$ \\
\hline SDSS J081456 & $24.3_{-16.4}^{+7.7}$ & $2409 \pm 61$ & $1438 \pm 32$ & $7.44_{-0.49}^{+0.12}$ & $0.59_{-0.30}^{+1.03}$ & $43.99 \pm 0.04$ & $42.15 \pm 0.03$ & $74.4 \pm 7.6$ \\
\hline SDSS J093922 & $11.9_{-6.3}^{+2.1}$ & $1209 \pm 16$ & $835 \pm 11$ & $6.53_{-0.33}^{+0.07}$ & $2.54_{-0.20}^{+0.71}$ & $44.07 \pm 0.04$ & $42.09 \pm 0.04$ & $53.0 \pm 6.7$ \\
\hline \multicolumn{9}{|c|}{ SEAMBH2014 } \\
\hline SDSS J075949 & $55.0_{-13.1}^{+17.0}$ & $1807 \pm 11$ & $1100 \pm 3$ & $7.54_{-0.12}^{+0.12}$ & $0.70_{-0.29}^{+0.29}$ & $44.20 \pm 0.03$ & $42.48 \pm 0.02$ & $97.5 \pm 9.1$ \\
\hline SDSS J080131 & $11.2_{-9.8}^{+14.8}$ & $1290 \pm 13$ & $800 \pm 5$ & $6.56_{-0.90}^{+0.37}$ & $2.29_{-0.80}^{+1.87}$ & $43.95 \pm 0.04$ & $41.96 \pm 0.05$ & $52.3 \pm 7.7$ \\
\hline SDSS J084533 & $15.2_{-6.3}^{+3.2}$ & $1243 \pm 13$ & $818 \pm 10$ & $6.66_{-0.23}^{+0.08}$ & $2.98_{-0.22}^{+0.52}$ & $44.54 \pm 0.04$ & $42.58 \pm 0.05$ & $55.9 \pm 7.5$ \\
\hline SDSS J085946 & $34.8_{-26.3}^{+19.2}$ & $1718 \pm 16$ & $1031 \pm 14$ & $7.30_{-0.61}^{+0.19}$ & $1.51_{-0.43}^{+1.27}$ & $44.41 \pm 0.03$ & $42.51 \pm 0.02$ & $63.1 \pm 5.2$ \\
\hline SDSS J102339 & $24.9_{-3.9}^{+19.8}$ & $1733 \pm 29$ & $1139 \pm 19$ & $7.16_{-0.08}^{+0.25}$ & $1.29_{-0.56}^{+0.20}$ & $44.09 \pm 0.03$ & $42.14 \pm 0.03$ & $57.0 \pm 5.9$ \\
\hline
\end{tabular}

Note. - All SEAMBH2012 measurements are taken from Paper III, but $5100 \AA$ Aluxes are from I and II, SEAMBH2013 from Paper IV, and SEAMBH2014 is the present paper. MCG +06-26-012 was selected as a super-Eddington candidate in SEAMBH2012 but later was identified to be a sub-Eddington accretor $(\dot{\mathscr{M}}=0.46)$; we discard it here.

As shown in Paper IV, the $\mathrm{H} \beta$ lags of the SEAMBH2013 sample were found to significantly deviate from the normal $R_{\mathrm{H} \beta}-L_{5100}$ relation, by a factor of a few. We plot the $R_{\mathrm{H} \beta}-$ $L_{5100}$ relation of all samples in Figure 3 . For sub-Eddington AGNs $(\dot{\mathscr{M}} \leq 3)$ in the direct scheme, $\log \left(R_{\mathrm{H} \beta} /\right.$ ltd $)=(1.54 \pm$ $0.03)+(0.53 \pm 0.03) \log \ell_{44}$, with an intrinsic scatter of 0.15 (see Paper IV). Using FITEXY, we have

$$
\begin{aligned}
& \log \left(R_{\mathrm{H} \beta} / \mathrm{ltd}\right)= \\
& \left\{\begin{array}{l}
(1.30 \pm 0.05)+(0.53 \pm 0.06) \log \ell_{44}(\dot{\mathscr{M}} \geq 3), \\
(1.44 \pm 0.03)+(0.49 \pm 0.03) \log \ell_{44} \quad(\text { for all } \dot{\mathscr{M}}),
\end{array}\right.
\end{aligned}
$$

with intrinsic scatters of $\sigma_{\text {in }}=(0.24,0.21)$. Clearly, the intrinsic scatter of SEAMBHs is much larger than the sample of sub-Eddington AGNs. In the averaged scheme, we have $\log \left(R_{\mathrm{H} \beta} /\right.$ ltd $)=(1.55 \pm 0.04)+(0.53 \pm 0.04) \log \ell_{44}$ for subEddington AGNs, with an intrinsic scatter of 0.16 (see Paper IV), and

$$
\begin{aligned}
& \log \left(R_{\mathrm{H} \beta} / \mathrm{ltd}\right)= \\
& \left\{\begin{array}{l}
(1.32 \pm 0.05)+(0.52 \pm 0.06) \log \ell_{44}(\dot{\mathscr{M}} \geq 3), \\
(1.44 \pm 0.03)+(0.49 \pm 0.03) \log \ell_{44} \quad(\text { for all } \dot{\mathscr{M}})
\end{array}\right.
\end{aligned}
$$

with intrinsic scatters of $\sigma_{\text {in }}=(0.22,0.21)$. The slope of the correlation for the SEAMBH sample is comparable to that of sub-Eddington AGNs, but the normalization is significantly different. It is clear that the SEAMBH sources increase the scatter considerably, especially over the limited luminosity range occupied by the new sources.

As in Paper IV, we also tested the correlation between $\mathrm{H} \beta$ lag and $\mathrm{H} \beta$ luminosity, namely, the $R_{\mathrm{H} \beta}-L_{\mathrm{H} \beta}$ relation. The scatter of the $R_{\mathrm{H} \beta}-L_{\mathrm{H} \beta}$ correlation is not smaller than that of the $R_{\mathrm{H} \beta}-L_{5100}$ correlation, and we do not consider it further.

\section{2. $\dot{\mathscr{M}}$-dependent BLR Size}

To test the dependence of the BLR size on accretion rate, we define a new parameter, $\Delta R_{\mathrm{H} \beta}=\log \left(R_{\mathrm{H} \beta} / R_{\mathrm{H} \beta, R-L}\right)$, that specifies the deviation of individual objects from the $R_{\mathrm{H} \beta}$ $L_{5100}$ relation of the subsample of $\dot{\mathscr{M}}<3.0$ sources (i.e., $R_{\mathrm{H} \beta, R-L}$ as given by Equations $4 \mathrm{~b}$ and $5 \mathrm{~b}$ for $\dot{\mathscr{M}}<3 \mathrm{AGNs}$ in Paper IV). The scatter of $\Delta R_{\mathrm{H} \beta}$ is calculated by $\sigma_{R_{\mathrm{H} \beta}}=$ $\left[\sum_{i}\left(\Delta R_{\mathrm{H} \beta, i}-\left\langle\Delta R_{\mathrm{H} \beta}\right\rangle\right)^{2} / N\right]^{1 / 2}$, where $N$ is the number of objects and $\left\langle\Delta R_{\mathrm{H} \beta}\right\rangle$ is the averaged value. Figure 3 provides $\Delta R_{\mathrm{H} \beta}$ plots for comparison.

Figure 4 shows $\Delta R_{\mathrm{H} \beta}$ versus $\dot{\mathscr{M}}$, as well as $\Delta R_{\mathrm{H} \beta}$ distributions for the $\dot{\mathscr{M}} \geq 3$ and $\dot{\mathscr{M}}<3$ subsamples in the di- 


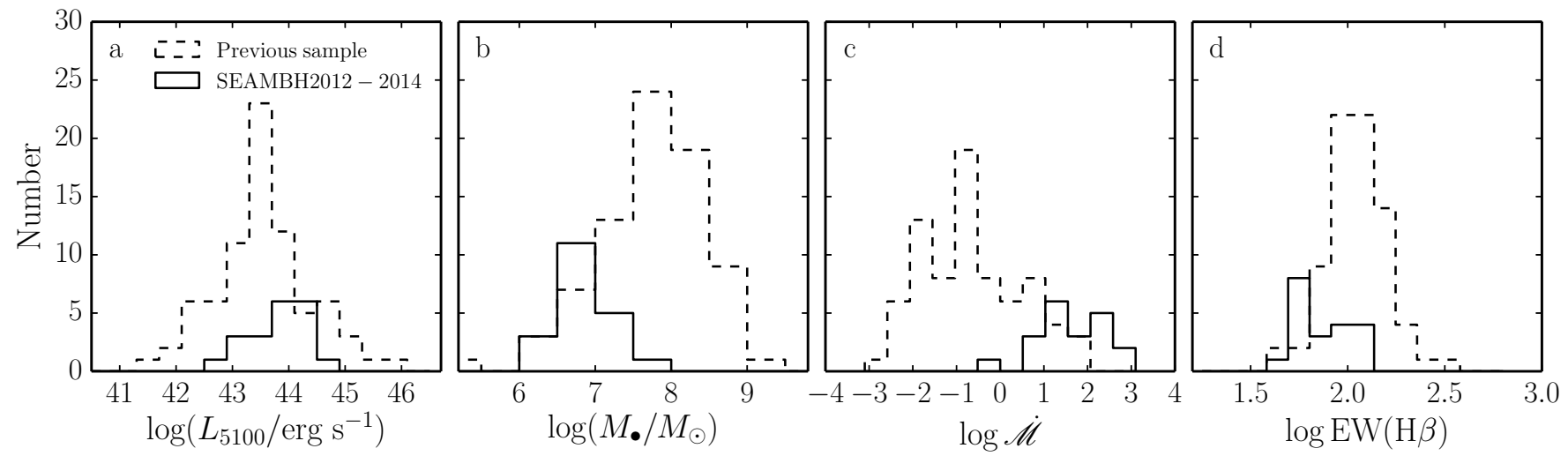

Figure 2. Distributions of $5100 \AA$ luminosity $\left(L_{5100}\right)$, BH mass $\left(M_{\bullet}\right)$, dimensionless accretion rate $(\dot{M})$, and equivalent width (EW) of all the mapped AGNs. These distributions show that the present sample of mapped AGNs is inhomogeneous. Only three luminous sources $\left(L_{5100} \gtrsim 10^{45} \mathrm{erg} \mathrm{s}^{-1}\right)$ have been mapped. The distribution of $\operatorname{EW}(\mathrm{H} \beta)$ in panel $d$ shows that the SEAMBH sample tends to have low $\operatorname{EW}(\mathrm{H} \beta)$.

rect (panels a and b) and averaged (panels $\mathrm{c}$ and d) schemes. A Kolmogorov-Smirnov (KS) test shows that the probability that the two subsamples are drawn from the same parent distributions is $p_{\mathrm{KS}}=0.00029$ for the direct scheme and $p_{\mathrm{KS}}=0.0094$ for the averaged scheme. This provides a strong indication that the main cause of deviation from the normal $R_{\mathrm{H} \beta}-L_{5100}$ relation is the extreme accretion rate. Thus, a single $R_{\mathrm{H} \beta}-L_{5100}$ relation for all AGNs is a poor approximation for a more complex situation in which both the luminosity and the accretion rate determine $R_{\mathrm{H} \beta}$. From the regression for $\dot{\mathscr{M}} \geq 3$ AGNs, we obtain the dependence of the deviations of $R_{\mathrm{H} \beta}$ from the $R_{\mathrm{H} \beta}-L_{5100}$ relation in Figure 4:

$$
\begin{aligned}
& \Delta R_{\mathrm{H} \beta}= \\
& \left\{\begin{array}{l}
(0.39 \pm 0.09)-(0.47 \pm 0.06) \log \dot{\mathscr{M}} \quad \text { (direct scheme), } \\
(0.34 \pm 0.09)-(0.42 \pm 0.07) \log \dot{\mathscr{M}} \quad \text { (averaged scheme), }
\end{array}\right.
\end{aligned}
$$

with $\sigma_{\text {in }}=(0.01,0.05)$, respectively. We have tested the above correlations also for $\dot{\mathscr{M}}<3$. The FITEXY regressions give slopes near 0 , with very large uncertainties: $\Delta R_{\mathrm{H} \beta} \propto$ $\dot{\mathscr{M}}^{-0.055 \pm 0.032}$ and $\Delta R_{\mathrm{H} \beta} \propto \dot{\mathscr{M}}^{-0.095 \pm 0.050}$ for Figure $4 \mathrm{a}$ and $4 \mathrm{c}$, respectively, implying that $\Delta R_{\mathrm{H} \beta}$ does not correlate with $\dot{\mathscr{M}}$ for the $\dot{\mathscr{M}}<3$ group. All this confirms that $\dot{\mathscr{M}}$ is an additional parameter that controls the $R_{\mathrm{H} \beta}-L_{5100}$ relation in AGNs with high accretion rates.

\section{A NEW SCALING RELATION FOR THE BLR}

We provide evidence $\mathrm{H} \beta$ lags depend on luminosity and accretion rate. There are a total of 28 SEAMBHs (including those discovered in other studies). We now have an opportunity to define a new scaling relation for the BLR, one that properly captures the behavior of sub-Eddington and superEddington AGNs. Considering the dependence of $\Delta R_{\mathrm{H} \beta} \propto$ $\dot{\mathscr{M}}^{-0.42}$ (Equation 6), a unified form of the new scaling law can take the form 13

$$
R_{\mathrm{H} \beta}=\alpha_{1} \ell_{44}^{\beta_{1}} \min \left[1,\left(\frac{\dot{\mathscr{M}}}{\dot{\mathscr{M}}_{\mathrm{c}}}\right)^{-\gamma_{1}}\right],
$$

where $\dot{\mathscr{M}}_{c}$ is to be determined by data. Equation (7) reduces to the normal $R_{\mathrm{H} \beta}-L_{5100}$ relation for sub-Eddington AGNs and to $R_{\mathrm{H} \beta}=\alpha_{1} \ell_{44}^{\beta_{1}}\left(\dot{\mathscr{M}} / \dot{\mathscr{M}}_{c}\right)^{-\gamma_{1}}$ for AGNs with $\dot{\mathscr{M}} \geq \dot{\mathscr{M}}_{c}$. There are four parameters to describe the new scaling relation, but only two $\left(\mathscr{\mathscr { M }}_{c}\right.$ and $\left.\gamma_{1}\right)$ are new due to the inclusion of accretion rates; the other two are mainly determined by subEddington AGNs. The critical value of $\dot{\mathscr{M}}_{c}$, which is different from the criterion of SEAMBHs, depends on the sample of SEAMBHs.

In order to determine the four parameters simultaneously, we define

$$
\chi^{2}=\frac{1}{N} \sum_{i=1}^{N} \frac{\left(R_{\mathrm{H} \beta}-R_{\mathrm{H} \beta}^{i}\right)^{2}}{\left(\Delta_{R_{\mathrm{H} \beta}}^{i}\right)^{2}},
$$

where $\Delta_{R_{\mathrm{H} \beta}}^{i}$ is the error bar of $R_{\mathrm{H} \beta}^{i}$. Minimizing $\chi^{2}$ among all the mapped AGNs and employing a bootstrap method, we have

$\alpha_{1}=29.6_{-2.8}^{+2.7} ; \quad \beta_{1}=0.56_{-0.03}^{+0.03} ; \quad \gamma_{1}=0.52_{-0.16}^{+0.33} ; \quad \dot{\mathscr{M}}_{c}=11.19_{-6.22}^{+2.29}$.

This new empirical relation has a scatter of 0.19 , smaller than the scatter $(0.26)$ of the normal $R_{\mathrm{H} \beta}-L_{5100}$ relation for all the mapped AGNs. The new scaling relation is plotted in Figure 5.

Equation (7) shows the dependence of the BLR size on accretion rates, but it cannot be directly applied to single-epoch spectra for BH mass without knowning $\dot{\mathscr{M}}$. Iteration of Equation (7) does not converge. The reason is due to the fact that larger $\dot{\mathscr{M}}$ leads to smaller $R_{\mathrm{H} \beta}$ and higher $\dot{\mathscr{M}}$, implying that the iteration from Equation (1) does not converge. Du et al. (2016b) devised a new method to determine $\ddot{\mathscr{M}}$ from singleepoch spectra. Beginning with the seminal work of Boroson

${ }^{13}$ We have tried $R_{\mathrm{H} \beta}=\alpha_{1} \ell_{44}^{\beta_{1}}\left[1+\left(\dot{\mathscr{M}} / \dot{\mathscr{M}}_{c}\right)^{\gamma_{1}}\right]^{\delta_{1}}$, which is continuous for the transition from sub- to super-Eddington sources. The fitting also yields a very rapid transition at $\dot{\mathscr{M}}_{\mathrm{c}} \sim 10$, with $\gamma_{1}=0.025$ and $\delta_{1}=21.02$ (the present sample is still dominated by sub-Eddington AGNs, with a ratio of 35/63). We prefer the form given by Equation (7). 

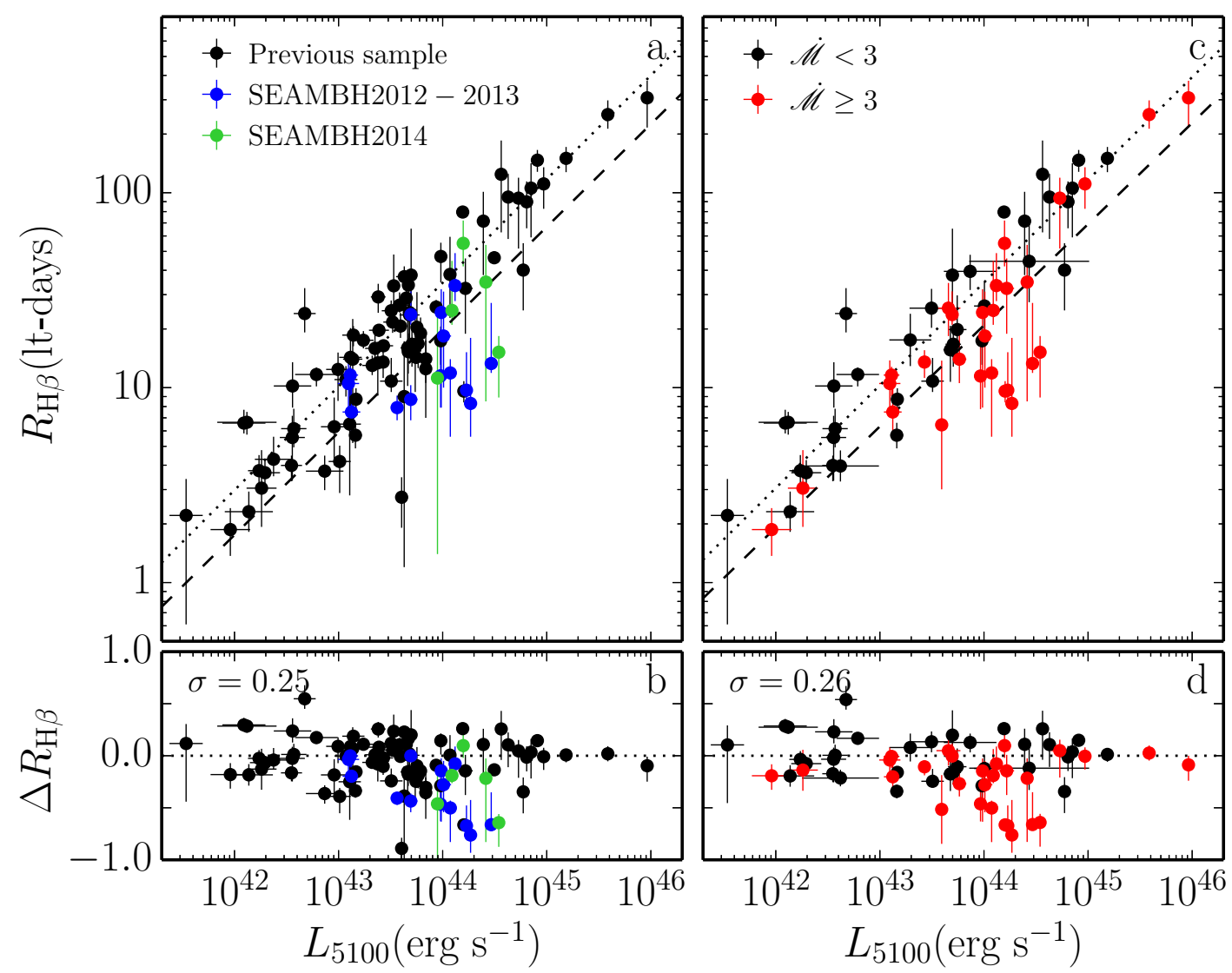

Figure 3. The $R_{\mathrm{H} \beta}-L_{5100}$ plot for all mapped AGNs. Left panel shows multiple-RM results as individual points, whereas the right panel shows the averaged results of AGNs with multiple-RM measurements. The dotted line is the regression of $R_{\mathrm{H} \beta}-L_{5100}$ relation for $\dot{\mathscr{M}}<3$ AGNs (Equation 4); the dashed line is the regression for the $\dot{\mathscr{M}} \geq 3$ objects. The scatter (standard deviations) of $\Delta R_{\mathrm{H} \beta}$ is given in the upper left corner of Panels $b$ and $d$.

\& Green (1992), it has been well-known that $\mathcal{R}_{\mathrm{Fe}} \equiv F_{\mathrm{FeII}} / F_{\mathrm{H} \beta}$, the flux ratio of broad optical $\mathrm{Fe}$ II to $\mathrm{H} \beta$, correlates strongly with Eddington ratio (Sulentic et al. 2000; Shen \& Ho 2014). At the same time, the shape of broad $\mathrm{H} \beta$, as parameterized by $\mathcal{D}_{\mathrm{H} \beta}=\mathrm{FWHM} / \sigma_{\mathrm{H} \beta}$, where $\sigma_{\mathrm{H} \beta}$ is the line dispersion, also correlates with Eddington ratio (Collin et al. 2006). Combining the two produces produces a strong bivariate correlation, which we call the fundamental plane of the BLR, of the form

$$
\log \dot{\mathscr{M}}=\alpha_{2}+\beta_{2} \mathcal{D}_{\mathrm{H} \beta}+\gamma_{2} \mathcal{R}_{\mathrm{Fe}},
$$

where

$$
\alpha_{2}=2.47 \pm 0.34 ; \beta_{2}=-1.59 \pm 0.14 ; \gamma_{2}=1.34 \pm 0.20 \text {. }
$$

\section{DISCUSSION}

\subsection{Normalized BLR Sizes}

In order to explore the relation between BLR size and accretion rate, we define a dimensionless radius for the BLR, $r_{\mathrm{H} \beta}=R_{\mathrm{H} \beta} / R_{\mathrm{g}}$, where $R_{\mathrm{g}}=1.5 \times 10^{12} m_{7} \mathrm{~cm}$ is the gravitational radius. As in Paper IV, we insert Equation (3) into $r_{\mathrm{H} \beta}$ to replace $\ell_{44}$, to obtain $r_{\mathrm{H} \beta}=1.9 \times 10^{4} \dot{\mathscr{M}}^{0.35} m_{7}^{-0.29}$. This relation implies that $r_{\mathrm{H} \beta}$ increases with accretion rates as $r_{\mathrm{H} \beta} \propto \dot{\mathscr{M}}^{0.35}$ for sub-Eddington AGNs, whereas in SEAMBHs $r_{\mathrm{H} \beta} \propto \dot{\mathscr{M}}^{0.29 \pm 0.08}$ (as shown in Figure $6 a$ ) and $r_{\mathrm{H} \beta}$ and tends toward a maximum saturated value of $r_{\mathrm{H} \beta}^{\max }=f_{\mathrm{BLR}}^{-1}\left(c / V_{\min }\right)^{2}=$ $9 \times 10^{4} f_{\mathrm{BLR}}^{-1} V_{\min , 3}^{-2}$, where $V_{\min , 3}=V_{\min } / 10^{3} \mathrm{~km} \mathrm{~s}^{-1}$ is the minimum velocity width of $\mathrm{H} \beta$ (see Equation 15 in Paper IV). We note that the minimum observed FWHM values of $\mathrm{H} \alpha$ (which is comparable to $\mathrm{H} \beta$ ) is $\sim 10^{3} \mathrm{~km} \mathrm{~s}^{-1}$ among low-mass AGNs (Greene \& Ho 2007; Ho \& Kim 2016). Indeed, this limit is consistent with the saturation trend of $r_{\mathrm{H} \beta}$ (Figure $6 a$ ).

We note that the relatively large scatter in Figure $6 a$ is mostly due to the uncertainties in BH mass. In order to better understand the relation between the BLR and the central engine, we define, as in Paper IV, the parameter $Y=m_{7}^{0.29} r_{\mathrm{H} \beta}$, which reduces to

$$
Y=1.9 \times 10^{4} \dot{\mathscr{M}}^{0.35}
$$

We would like to point out that Equation (12) describes the coupled system of the BLR and the accretion disks. It is therefore expected that $Y$ is a synthetic parameter describing the photoionization process including ionizing sources.

It is easy to observationally test Equation (12) using RM results. Figure $6 b$ plots $Y$ versus $\ddot{\mathscr{M}}$. It is very clear that the observed data for objects with $\mathscr{\mathscr { M }}<3$ agree well with Equation (12). Furthermore, there is a clear saturation of $Y$ for objects with $\dot{\mathscr{M}} \geq 3$ objects. All these results strengthen the conclusions drawn in Paper IV. As in that work, we define an empirical relation

$$
Y=Y_{\text {sat }} \min \left[1,\left(\frac{\dot{\mathscr{M}}}{\dot{\mathscr{M}}_{b}}\right)^{b}\right],
$$



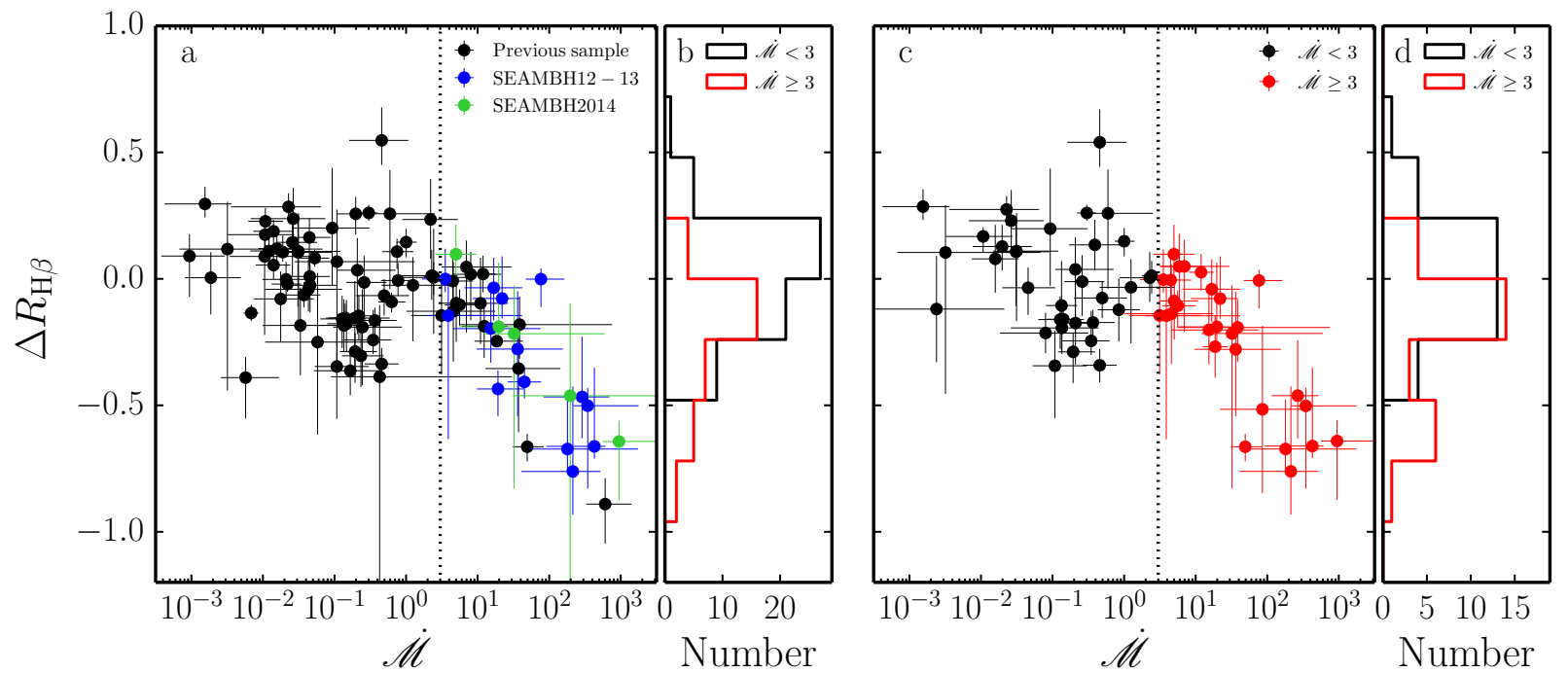

Figure 4. The deviation of $\mathrm{H} \beta$ lags from the normal $R_{\mathrm{H} \beta}-L_{5100}$ relation for sub-Eddington AGNs. Panel $a$ shows the sample of all mapped AGNs (the repeatedly monitored AGNs are regarded as individual ones). Panel $b$ gives the distribution of low- and high-accretion objects. Panels $c$ and $d$ are the same plots but for the average scheme. Sub-Eddington AGNs show a random distributions, but SEAMBHs correlate with accretion rates. We note that $M_{\bullet}$ and $\mathscr{M}$ are calculated in exactly the same way for all objects, as indicated in Table 7 in Paper IV (i.e. using Equations 2 and 3).

where

$$
Y_{\text {sat }}=\left(3.5_{-0.5}^{+0.6}\right) \times 10^{4}, \quad \dot{\mathscr{M}}_{b}=15.6_{-9.1}^{+22.0}, \quad b=0.27_{-0.04}^{+0.04}
$$

In fact, we can get $Y$ by inserting Equation (3) into (7), and find that it is in agreement with Equation (13). From the saturated $-Y$, we have the maximum value of

$$
r_{\mathrm{H} \beta \text {, sat }}=\left(3.5_{-0.5}^{+0.6}\right) \times 10^{4} m_{7}^{-0.29} \text { or } R_{\mathrm{H} \beta, \text { sat }}=\left(19.9_{-2.8}^{+3.4}\right) m_{7}^{0.71} \mathrm{ltd} \text {. }
$$

This result provides a strong constraint on theoretical models of super-Eddington accretion onto BHs.

\subsection{The Shortened Lags}

The shortened $\mathrm{H} \beta$ lags is the strongest distinguishing characteristic so far identified between super- and subEddington AGNs. Two factors may lead to shortened lags for SEAMBHs. First, Wang et al. (2014c) showed that, in the Shakura-Sunyaev regime, retrograde accretion onto a $\mathrm{BH}$ can lead to shorter $\mathrm{H} \beta$ lags. The reason is due to the suppression of ionizing photons in retrograde accretion compared with prograde accretion. The second factor stems from selfshadowing effects of the inner part of slim disks (e.g., Li et al. 2010), which efficiently lower the ionizing flux received by the BLR (Wang et al. 2014c). When $\mathscr{M}$ increases, the ratio of the disk height to disk radius increases due to radiation pressure; the radiation field becomes anisotropic (much stronger than the factor of $\cos i$ ) due to the optically thick funnel of the inner part of the slim disk. In principle, the radiation from a slim disk saturates $(\propto \ln \dot{\mathscr{M}})$, and the total ionizing luminosity slightly increases with accretion rate, but the selfshadowing effects efficiently suppress the ionizing flux to the BLR clouds. For face-on disks of type 1 AGNs, observers receive the intrinsic luminosity. If the ionization parameter is constant, the ionization front will significantly shrink, and hence the $\mathrm{H} \beta$ lag is shortened in SEAMBHs compared with sub-Eddington AGNs of the same luminosity.

The shortened $\mathrm{H} \beta$ lag observed in SEAMBHs cannot be caused by retrograde accretion. However, the strong dependence on accretion rate of the deviation from the standard lagluminosity relation implies that the properties of the ionizing sources are somehow different from those in sub-Eddington AGNs. According to the standard photoionization theory, the observed $R_{\mathrm{H} \beta} \propto L_{5100}^{1 / 2}$ relation can be explained if $L_{5100} \propto L_{\text {ion }}$ and $Q_{c}=U n_{e} \bar{\epsilon}$ is constant, where $U=L_{\mathrm{ion}} / 4 \pi R_{\mathrm{H} \beta}^{2} c n_{e} \bar{\epsilon}, L_{\mathrm{ion}}$ is the ionizing luminosity, $n_{e}$ is gas density of BLR clouds, and $\bar{\epsilon}$ is the average energy of the ionizing photons (Bentz et al. 2013). The relation $L_{5100} \propto L_{\text {ion }}$ holds for sub-Eddington AGNs, and the constancy of $Q_{c}$ is determined by the clouds themselves. $Q_{c}$ is not expected to vary greatly as a function of Eddington ratio. Therefore,

$$
R_{\mathrm{H} \beta}=\frac{L_{\mathrm{ion}}^{1 / 2}}{Q_{c}}=\mathcal{S} R_{\mathrm{H} \beta}^{0},
$$

where the factor $\mathcal{S}=\left(L_{\text {ion }} / L_{\text {ion, } 0}\right)^{1 / 2}$ describes the anisotropy of the ionizing radiation field, $L_{\text {ion }}$ is the shadowed ionizing luminosity received by the BLR clouds, and $R_{\mathrm{H} \beta}^{0}$ is the BLR radius corresponding to $L_{\mathrm{ion}, 0}$, the unshadowed luminosity. Based on the classical model of slim disks, Wang et al. (2014c) showed that, for a given accretion rate, $\mathcal{S}$ strongly depends on the orientation of the clouds relative to the disk, and that it range from 1 to a few tens. Therefore, the reduction of the $\mathrm{H} \beta$ lag can, in principle, reach up to a factor of a few, even 10 , as observed.

Furthermore, the saturated- $Y$ implies that the ionizing luminosity received by the BLR clouds gets saturated. The theory of super-Eddington accretion onto BHs is still controversial. Although extensive comparison with models is beyond the scope of this paper, we briefly discuss the implications of the current observations to the theory. Two analytical models, which reach diametrically extreme opposite conclusions, have been proposed. Abramowicz et al. (1988) suggested a model characterized by fast radial motion with sub-Keplerian rotation and strong photon-trapping. Both the shortened lags and saturated $-Y$ may be caused by self-shadowing effects and saturated radiation from a slim disk. Both features are expected from the Abramowicz et al. model (Wang et al. 2014b). On the other hand, photon-bubble instabilities may govern the disk structure and lead to very high radiative efficiency (Gam- 
mie 1998). If super-Eddington accretion can radiate as much as $L / L_{\text {Edd }} \gtrsim 470 m_{7}^{6 / 5}$ (Equation 14 in Begelman 2002), the disk remains geometrically thin. In such an extreme situation, self-shadowing effects are minimal, $\mathrm{H} \beta$ lags should not be reduced, and $Y$-saturation disappears.

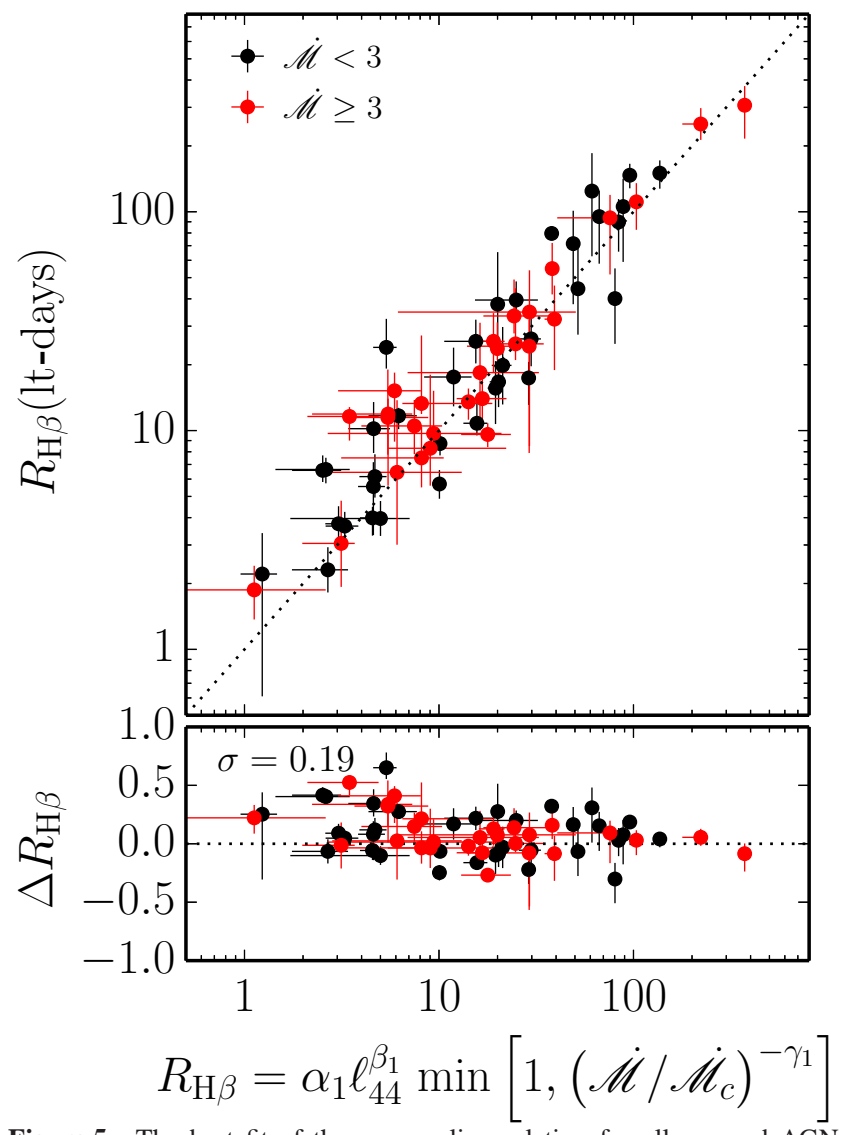

Figure 5. The best fit of the new scaling relation for all mapped AGNs. We find that $\alpha_{1}=\left(29.6_{-2.8}^{+2.7}\right) \mathrm{lt}-\mathrm{d}, \beta_{1}=0.56_{-0.03}^{+0.03}, \gamma_{1}=0.52_{-0.16}^{+0.33}$ and $\dot{M}_{c}=$ $11.19_{-6.22}^{+2.29}$. The scatter of the BLR size is greatly reduced to $\sigma=0.19$.

Recent numerical simulations that incorporate outflows (e.g., Jiang et al. 2014) and relativistic jets (Sadowski et al. 2015) also suggest that super-Eddington accretion flows can maintain a high radiative efficiency. However, most AGNs with high accretion rates are radio-quiet (Ho 2002; Greene \& Ho 2006), in apparent contradiction with the numerical simulation predictions. Furthermore, evidence for $Y$-saturation also does not support the models with high radiative efficiency. Recent modifications of the classical slim disk model that include photo-trapping appear promising (e.g., Cao \& Gu 2015; Sadowski et al. 2014), but the situation is far from settled. Whatever the outcome, the results from our observations provide crucial empirical constraints on the models.

\subsection{Inclination Effects on $\dot{\mathscr{M}}$}

If the BLR is flattened, its inclination angle to the observer will influence $M_{\bullet}$, and hence $\dot{\mathscr{M}}$ (see Equation 3 ). To zeroorder approximation, the observed width of the broad emission lines follow

$$
\Delta V_{\mathrm{obs}} \approx\left[\left(\frac{H_{\mathrm{BLR}}}{R}\right)^{2}+\sin ^{2} i\right]^{1 / 2} V_{\mathrm{K}},
$$

where $V_{\mathrm{K}}$ is the Keplerian velocity and $H_{\mathrm{BLR}}$ is the height of the flatten BLR (e.g., Collin et al. 2006). For a geometrically thin BLR, $H_{\mathrm{BLR}} / R \ll 1, \Delta V_{\mathrm{obs}} \approx V_{\mathrm{K}} \sin i$, and hence $\dot{\mathscr{M}} \propto$ $(\sin i)^{-4}$, which is extremely sensitive to the inclination can be severely overestimated for low inclinations. On the other hand, many arguments (e.g., Goad \& Korista 2014) support $H_{\mathrm{BLR}} / R \lesssim 1$, and the inclination angle significantly influences $M$ • only for $\sin i \gtrsim H_{\mathrm{BLR}} / R$. Currently, the values of $H_{\mathrm{BLR}} / R$ are difficult to estimate, but detailed modelling of RM data suggests $H_{\mathrm{BLR}} / R \sim 1$ (Li et al. 2013; Pancoast et al. 2014). If true, this implies that the BLR is not very flattened, and hence the inclination angle only has a minimal influence on $M_{\bullet}$ and $\dot{\mathscr{M}}$.

\subsection{Comparison with Previous Campaigns}

The objects in our SEAMBH sample are very similar to NLS1s. As previous RM AGN samples include NLS1s, why have previous studies not noticed that NLS1s deviate from the $R_{\mathrm{H} \beta}-L_{5100}$ relation (e.g., Figure 2 in Bentz 2011)? We believe that the reason is two-fold. First, the number of NLS1s included in previous RM campaigns was quite limited (Denney et al. 2009, 2010; Bentz et al. 2008, 2009; see summary in Bentz 2011). The level of optical variability in NLS1s is generally very low (Klimek et al. 2004), and many previous attempts at RM have proved to be unsuccessful (e.g., Giannuzzo \& Stirpe 1996; Giannuzzo et al. 1999). Second, not all NLS1s are necessarily highly accreting. Our SEAMBH sample was selected to have high accretion rates (see $\dot{\mathscr{M}}$ listed in Table 7 of Paper IV), generally higher than that of typical NLS1s previously studied successfully through RM. As discussed in Wang et al. (2014b) and in Paper IV, high accretion rates lead to anisotropic ionizing radiation, which may explain the shortened BLR lags.

\subsection{SEAMBHs as Standard Candles}

Once its discovery, quasars as the brightest celestial objects in the Universe had been suggested for cosmology (Sandage 1965; Hoyle \& Burbidge 1966; Longair \& Scheuer 1967; Schmidt 1968; Bahcall \& Hills 1973; Burbidge \& O’Dell 1973; Baldwin et al. 1978). Unfortunately, the diversity of observed quasars made these early attempts elusive. After five decades since its discovery, quasars are much well understood: accretion onto supermassive black holes is powering the giant radiation, in particular, the $\mathrm{BH}$ mass can be reliably measured. Quasars as the most powerful emitters renewed interests for cosmology in several independent ways: 1) the normal $R_{\mathrm{H} \beta}-L_{5100}$ relation (Horn et al. 2003; Watson et al. 2011; Czerny et al. 2013); 2) the linear relation between $\mathrm{BH}$ mass and luminosity in super-Eddington quasars (Wang et al. 2013; Paper-II); 3) Eddington AGNs selected by eigenvector 1 (Marziani \& Sulentic 2014); 4) X-ray variabilities (La Franca et al. 2014) and 5) $\alpha_{\mathrm{OX}}-L_{X}$ relation (Risaliti \& Lusso 2015). These parallel methods will be justified for cosmology by their feasibility of experiment periods and measurement accuracy.

The strength of SEAMBHs makes its application more convenient for cosmology. Selection of SEAMBHs only depends on single epoch spectra through the fundamental plane (Equation 10). BH mass can be estimated by the new scaling relation (Equation 7). We will apply the scheme outlined by Wang et al. (2014a) to the sample of selected SEAMBHs for cosmology in a statistical way (in preparation). On the other hand, the shortened $\mathrm{H} \beta$ lags greatly reduce monitoring 

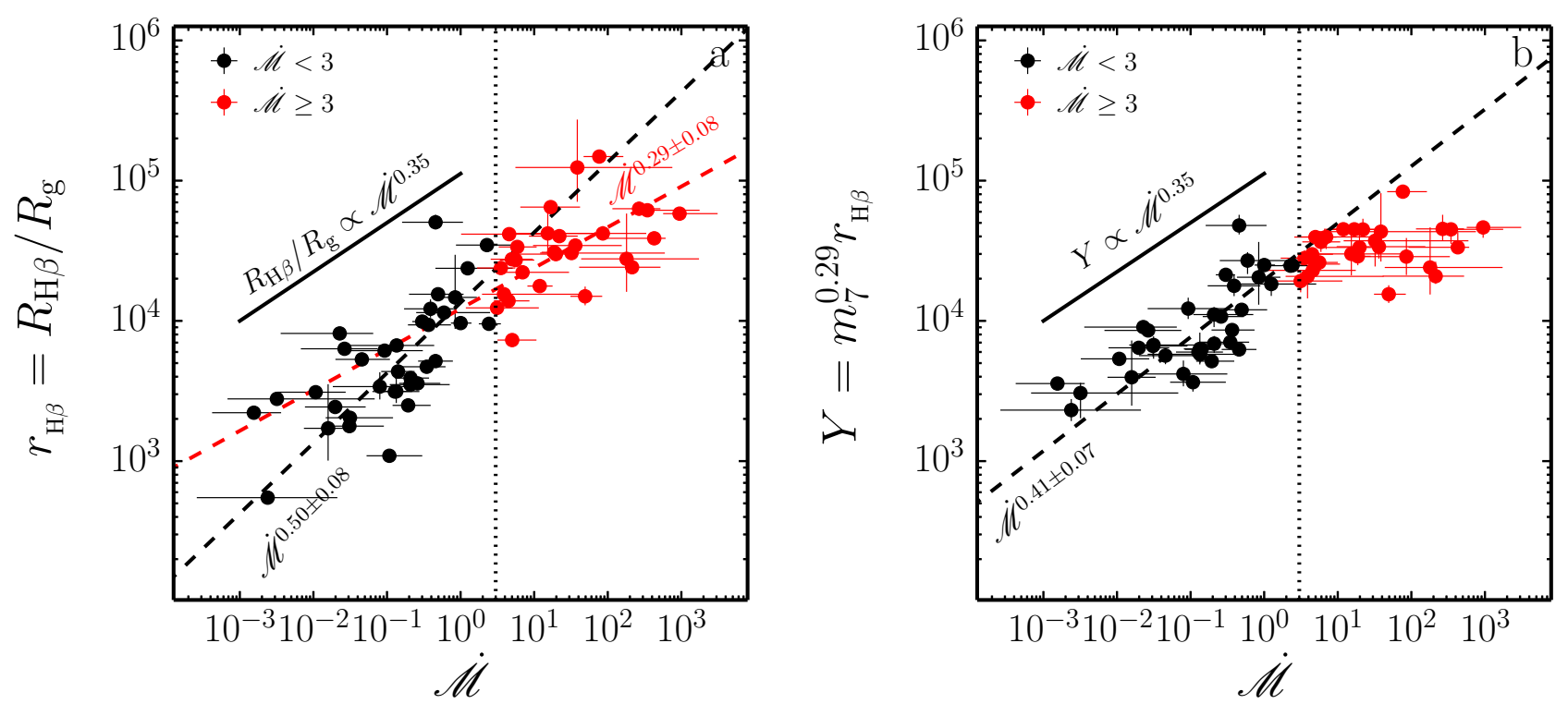

Figure 6. The dimensionless BLR radius and $Y$-parameter versus accretion rate $\dot{\mathscr{M}}$. There is a trend of saturation of $r_{\mathrm{H} \beta}$ as shown in panel $a$, but it is caused by the scatter of BH mass. Panel $b$ shows much a tighter relation and unambiguous saturation of the $Y$-parameter. We should point out that NGC 7469 in this plot has been revised compared with Figure 5 in Paper IV, using the latest observation from Peterson et al. (2014).

periods if SEAMBHs are applied as standard candles, in particular, the reduction of lags govern by super-Eddington accretion can cancel the cosmological dilltion factor of $(1+z)$. Otherwise, the monitoring periods of sub-Eddington AGNs should be extended by the same factor of $(1+z)$ for measurements of $\mathrm{H} \beta$ lags. Such a campaign of using the normal $R_{\mathrm{H} \beta}-L_{5100}$ relation for cosmology will last for a couple of years, even 10 years for bright high $-z$ quasars. Similarly to extension of the $R_{\mathrm{H} \beta}-L_{5100}$ relation to Mg II- and C IV-lines (Vestergaard \& Peterson 2006), we can extend Equation (7) to $\mathrm{Mg}$ II and C IV lines for the scaling relations with luminosity as $R_{\mathrm{MgII}}\left(L_{3000}, \dot{\mathscr{M}}\right)$ and $R_{\mathrm{CIV}}\left(L_{3000}, \dot{\mathscr{M}}\right)$, respectively, where $R_{\mathrm{MgII}}$ and $R_{\mathrm{CIV}}$ are sizes of the $\mathrm{Mg}$ II and C IV regions, and $L_{3000}$ is the $3000 \AA$ luminosity. Such extended relations conveniently allow us to investigate cosmology by making use of large samples of high $-z$ quasars without time-consuming RM campaigns. It is urgent for us to make use of kinds of standard candles to test the growing evidence for dynamical dark energy (e.g., Zhao et al. 2012; Ade et al. 2015).

\section{CONCLUSIONS}

We present the results of the third year of reverberation mapping of super-Eddington accreting massive black holes (SEAMBHs). $\mathrm{H} \beta$ lags of five new SEAMBHs have been detected. Similar to the SEAMBH2012 and SEAMBH2013 samples, we find that the SEAMBH2014 objects generally have shorter $\mathrm{H} \beta$ lags than the normal $R_{\mathrm{H} \beta}-L_{5100}$ relation, by a factor of a few. In total, we have detected $\mathrm{H} \beta$ lags for 18 SEAMBHs from this project, which have accretion rates from $\dot{\mathscr{M}} \sim 10$ to $\lesssim 10^{3}$. The entire SEAMBH sample allows us to establish a new scaling relation for the BLR size, which depends not only on luminosity but also on accretion rate. The new relation, applicable over a wide range of accretion rates from $\dot{\mathscr{M}} \approx 10^{-3}$ to $10^{3}$, is given by $R_{\mathrm{H} \beta}=\alpha_{1} \ell_{44}^{\beta_{1}} \min \left[1,\left(\dot{\mathscr{M}} / \dot{\mathscr{M}}_{c}\right)^{-\gamma_{1}}\right]$, where $\ell_{44}=$ $L_{5100} / 10^{44} \mathrm{erg} \mathrm{s}^{-1}$ is $5100 \AA$ continuum luminosity, and coefficients of $\alpha_{1}=\left(29.6_{-2.8}^{+2.7}\right) \mathrm{lt}-\mathrm{d}, \beta_{1}=0.56_{-0.03}^{+0.03}, \gamma_{1}=0.52_{-0.16}^{+0.33}$ and $\dot{\mathscr{M}}_{c}=11.19_{-6.22}^{+2.29}$.
We thank an anonymous referee for critical comments that helped to improve the paper. We acknowledge the support of the staff of the Lijiang $2.4 \mathrm{~m}$ telescope. Funding for the telescope has been provided by CAS and the People's Government of Yunnan Province. This research is supported by the Strategic Priority Research Program - The Emergence of Cosmological Structures of the Chinese Academy of Sciences, Grant No. XDB09000000, by NSFC grants NSFC$11173023,-11133006,-11373024,-11503026,-11233003$ and -11473002, and a NSFC-CAS joint key grant U1431228, and by the CAS Key Research Program through KJZDEW-M06, and by a China-Israel project through NSFC11361140347.

\section{APPENDIX}

\section{A. VALIDITY OF EQUATION (3)}

The validity of Equation (3) can be justified for application to SEAMBHs. Solutions of slim disks are transonic and usually given only by numerical calculations (Abramowicz et al. 1988). When the accretion rate of the disk is high enough, the complicated structure of the disk reduces to a self-similar, analytical form (Wang \& Zhou 1999). Using the self-similar solutions (Wang \& Zhou 1999; Wang et al. 1999), we obtained the radius of disk region emitting optical (5100 $\AA$ ) photons,

$$
\frac{R_{5100}}{R_{\mathrm{Sch}}} \approx 4.3 \times 10^{3} m_{7}^{-1 / 2},
$$

and the photon-trapping radius

$$
\frac{R_{\text {trap }}}{R_{\mathrm{Sch}}} \approx 144\left(\frac{\dot{\mathscr{M}}}{10^{2}}\right) .
$$

We used the blackbody relation $k T_{\text {eff }}=h c / \lambda$, where $k$ is the Boltzmann constant, $T_{\text {eff }}$ is the effective temperature of the disk surface, $h$ is the Planck constant, and $R_{\mathrm{Sch}}=3.0 \times$ $10^{12} m_{7} \mathrm{~cm}$ is the Schwartzschild radius. Equation (3) holds provided $R_{5100} \gtrsim R_{\text {trap }}$, namely

$$
\dot{\mathscr{M}} \lesssim 3 \times 10^{3} m_{7}^{-1 / 2} .
$$



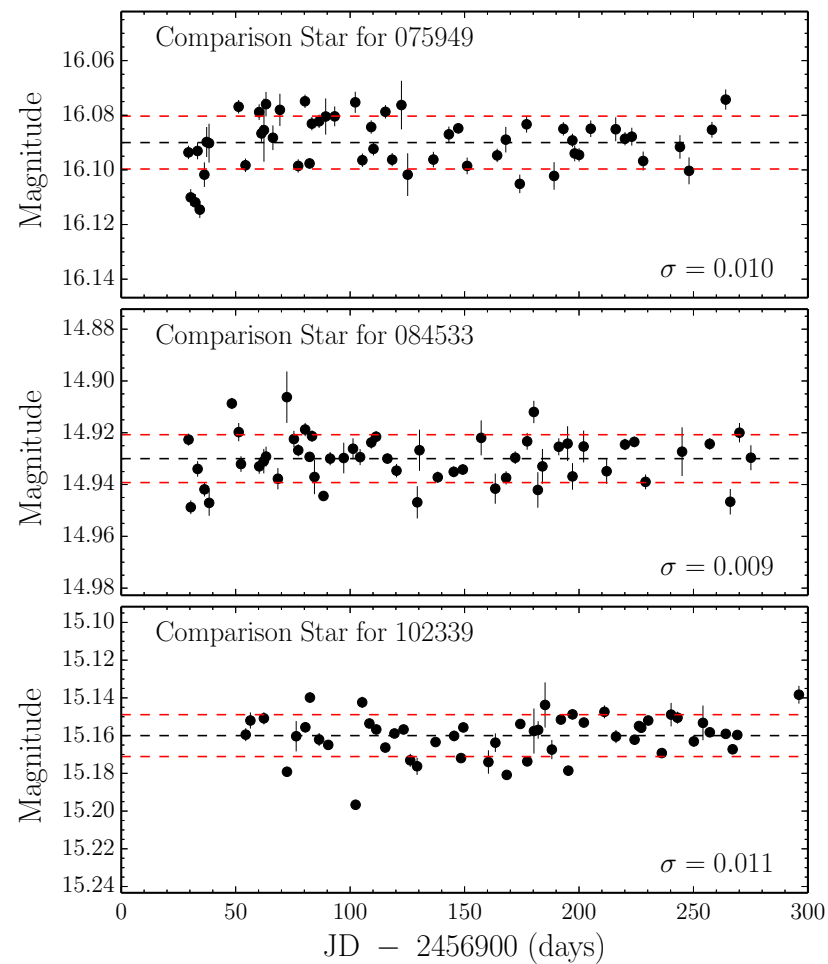

Figure 7. Photometric light curves of comparison stars in the slit.

In this regime, optical radiation is not influenced by photontrapping effects. We would also like to point out that the $\mathrm{BH}$ spin only affects emission from the innermost regions of the accretion disk rather than the regions emitting $5100 \AA$ photons. In the present campaign, no SEAMBH so far has been found to exceed this critical value. Beyond this critical value of accretion rate, optical photons are trapped by the accretion flow. We call this the hyper-accretion regime.

Here the cited $10^{-2}$ below Equation (3) is not a strict value of the ADAF threshold since it depends on several factors, such as viscosity and outer boundary conditions. There are a few mapped AGNs with $\dot{\mathscr{M}} \lesssim 10^{-2}$ (NGC 4151, NGC 5273, 3C 390.3 and NGC 5548; see Table 7 in Paper IV), but we do not discuss them in this paper because they do not influence our conclusions.

Recently, reprocessing of X-rays (e.g., Frank et al. 2002; Cackett et al. 2007) has been found to play an important role in explaining the variability properties of NGC 5548 (e.g., Fausnaugh et al. 2015). The fraction of X-ray emission to the bolometric luminosity strongly anti-correlates with the Eddington ratio (see Figure 1 in Wang et al. 2004). This result is usually interpreted to mean that the hot corona becomes weaker with increasing accretion rate, as a result of more efficiently cooling of the corona by UV and optical photons from the cold disk. This suggests that AGNs with high accretion rates will have less reprocessed emission, such that Equation (3) would be more robust in SEAMBHs.

\section{B. LIGHT CURVES OF COMPARISON STARS}

In order to avoid selecting variable stars as comparison stars, we examined their variability. To test the invariance of the comparison stars used in our spectroscopic observation, we performed differential photometry by comparing them with other stars in the same field. We typically use six stars for the differential photometry. The light curves of the stars are

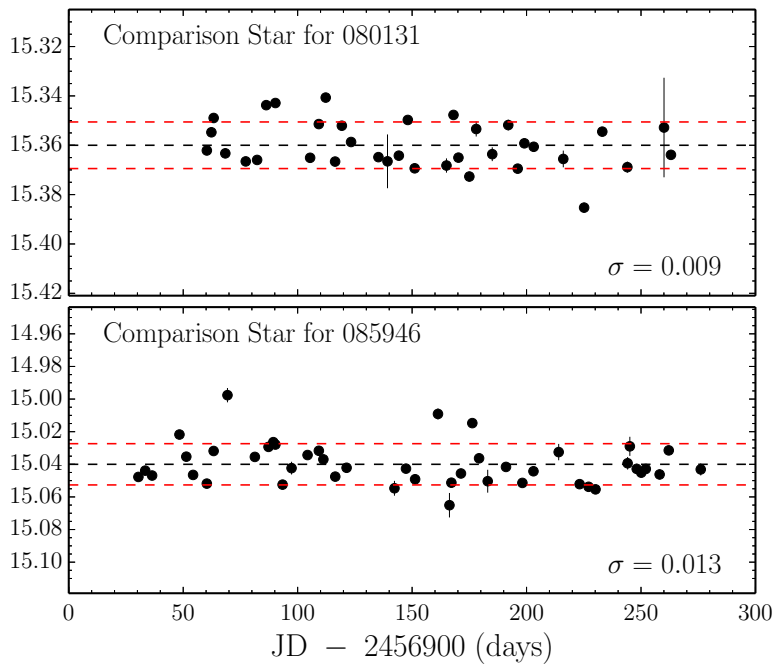

shown in Figure 7. On average, the standard deviations in the light curves of the comparison stars are $1 \%$. This guarantees that they can be used as standards for spectral calibration. Figure 7 shows the light curves of the comparison stars for each SEAMBH targets.

\section{AVERAGED AND RMS SPECTRA}

The averaged and RMS spectra of the SEAMBH2014 sample are provided in this Appendix. Following the standard way, we calculated the averaged spectrum as

$$
\bar{F}_{\lambda}=\frac{1}{N} \sum_{i=1}^{N} F_{\lambda}^{i}
$$

and the RMS spectrum as

$$
S_{\lambda}=\left[\frac{1}{N} \sum_{i=1}^{N}\left(F_{\lambda}^{i}-\bar{F}_{\lambda}\right)^{2}\right]^{1 / 2},
$$

where $F_{\lambda}^{i}$ is the $i$-th observed spectrum and $N$ is the total number of observed spectra. They are shown in Figure 8. We note that both the averaged and RMS spectra are affected by the broadening effects of the $5^{\prime \prime}$-slit on the observed profiles. Using the Richards-Lucy iteration, we can correct the observed profiles (averaged and RMS) for velocity-resolved mapping, which will be carried out in a separate paper (Du et al. 2015c).

\section{NOTES ON INDIVIDUAL OBJECTS}

We briefly remark on individual objects for which $\mathrm{H} \beta$ lags have been successfully measured. We failed in getting lag measurements for the other five objects because either their flux variations are very small or the data sampling rate was inadequate.

J075949: The detected $\mathrm{H} \beta$ lag arises from two major peaks in the light curves. 

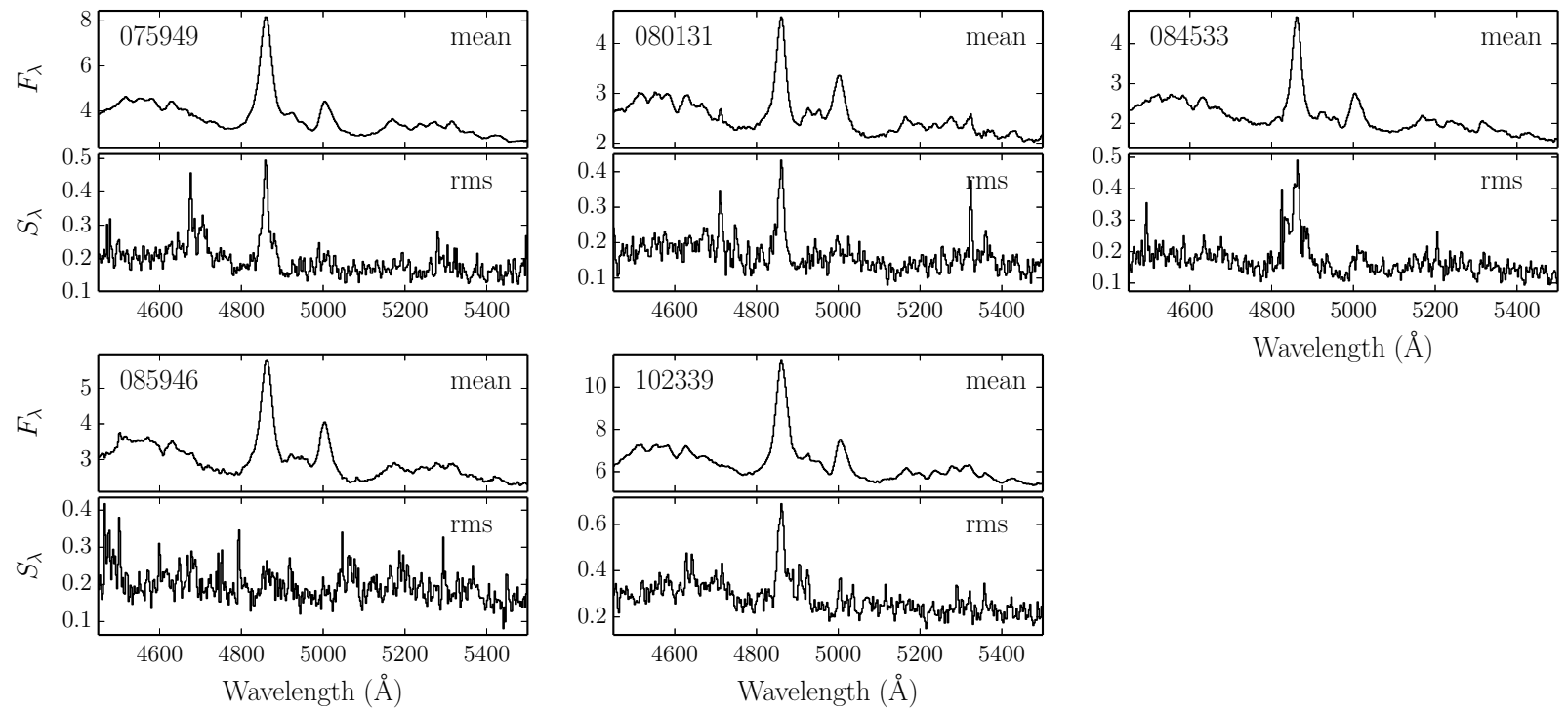

Wavelength $(\AA)$

Figure 8. The averaged and RMS spectra of the SEAMBH2014 sources. $S_{\lambda}$ and $\bar{F}_{\lambda}$ are in units of $10^{-16} \mathrm{erg} \mathrm{s}^{-1} \mathrm{~cm}^{-2-1}$.

J080131: The monitoring observations during 2013-2014 did not yield a well-determined $\mathrm{H} \beta$ lag because of the lack of $\mathrm{H} \beta$ response to the second continuum flare (see Paper IV). During 2013-2014, the first reverberation of $\mathrm{H} \beta$, which can be clearly seen during the first 70 days of the light curves, yields a very significant lag, as shown in the CCF with a restframe centroid lag of $11.5_{-3.6}^{+8.4}$ days (with a very high coefficient of $\left.r_{\max }=0.81\right)$. We monitored this object again in this observing season (Figure 1). We successfully measure $\tau_{\mathrm{H} \beta}=11.2_{-9.8}^{+14.8}$ days, consistent with last season's result.

J084533: Its continuum slightly decreased before being monitored for $\sim 70$ days, and steadily increased until $\sim 200$ days and then decreased again. Although the CCF has a very flat peak close to $\sim 0.9$, Monte Carlo simulations show that $\mathrm{H} \beta$ lag is around 20 days, which arises from the two peaks in the $\mathrm{H} \beta$ and $r^{\prime}$-band light curves.

J085946: The CCF peaks near 0.6, which results from the two major dips in the $\mathrm{H} \beta$ and $r^{\prime}$-band light curves. There are two peaks with roughly the same correlation coefficients around $\sim 20$ days and $\sim 70$ days in the observed frame, respectively. Considering the relatively poor data quality of this object, it is difficult to distinguish which is the true response. The centroid lag represents the average of these two peaks (responses), and its uncertainties cover the distribution (Figure 1) obtained in FR/RSS method. So, we use it in the analysis of main text.

J102339: The detected lag is from the dip feature around $\sim 150$ days in light curves.

\section{E. DESCRIPTION OF CCCD}

For multiple-peaked CCFs with similar correlation coefficients, it is ambiguous as to which peak should be used to calculate the final lag. In such cases, we use the CCCD to determine the lag. However, there are two approaches to calculate the centroid time lag in the CCF, as illustrated in Figure 9.

- Approach 1 calculates the centroid using all peaks above some criterion, such as $0.8 r_{\max }$.

- Approach 2 only uses the highest peak.
In Approach 1, the CCCD tends to be smoother than the CCPD (see Figure 1), whereas in in Approach 2 the CCCD and CCPD always have a similar distribution. We adopt Approach 1 in our analysis. If the CCF has two or even three peaks, the two approaches give different centroid lags. However, when the quality of the data is high and the CCF is unimodal, the two approaches yield the same results. It should be pointed out that Approach 2 is often employed in the literature.

\section{REFERENCES}

Abramowicz, M. A., Czerny, B., Lasota, J.-P., \& Szuszkiewicz, E. 1988, ApJ, 332, 646

Ade, P. A. R., Aghanim, N., Armitage-Caplan, C. et al. 2014, A\&A, 571, 16 Ade, P. A. R., Aghanim, N., Arnaud, M. et al. (Planck collaboration), 2015, arXiv: 1502.01590

Akritas, M. G. \& Bershady, M. A. 1996, ApJ, 470, 706

Arav, N., Barlow, T. A., Laor, A., \& Blandford, R. D. 1997, MNRAS, 288, 1015

Bahcall, J. N., Kozlovsky, B.-Z. \& Salpeter, E. E. 1972, ApJ, 171, 467

Bahcall, J. N. \& Hills, R. E. 1973, ApJ, 179, 699

Baldwin, J. A., Burke, W. L., Gaskell, C. M. \& Wampler, E. J. 1978, Nature, 273,431

Bardeen, J. M., Press, W. H. \& Teukolsky, S. A. 1972, ApJ, 178, 347

Barth, A. J., Bennert, V. N., Canalizo, G. et al. 2015, ApJS, 217, 26

Barth, A. J., Pancoast, A., Bennert, V. N. et al. 2013, ApJ, 769, 128

Begelman, M. C. 2002, ApJ, 568, L97

Beloborodov, A. M. 1998, MNRAS, 297, 739

Bentz, M. C. 2011, in Narrow-Line Seyfert 1 Galaxies and Their Place in the Universe, ed. L. Foschini et al., p33

Bentz, M. C., Walsh, J. L., Barth, A. J. et al. 2008, ApJ, 689, L21

Bentz, M. C. et al. 2009, ApJ, 705, 199

Bentz, M. C., Denney, K. D., Grier, C. J. et al. 2013, ApJ, 767, 149

Bentz, M. C., Horenstein, D., Bazhaw, C. et al. 2014, ApJ, 796, 8

Bentz, M. C., Walsh, J. L., Barth, A. J. et al. 2009, ApJ, 705, 199

Blandford, R. D. \& McKee, C. F. 1982, ApJ, 255, 419

Boroson, T. A. \& Green, R. F. 1992, ApJS, 80, 109

Brocksopp, C., Starling, R. L. C., Schady, P. et al. 2006, MNRAS, 366, 953

Burbidge, G. R. \& O'dell, S. L. 1973, ApJ, 183, 759

Clavel, J., Reichert, G. A., Alloin, D. et al. 1991, ApJ, 366, 64

Capellupo, D. M., Netzer, H., Lira, P. et al. 2015, MNRAS, 446, 3427

Cao, X. \& Gu, W.-M. 2015, MNRAS, 448, 3514

Collier, S. J., Horne, K., Kapsi, S. et al. 1998, ApJ, 500, 162

Collin, S., Boisson, C., Mouchet, M. et al. 2002, A\&A, 388, 771

Collin, S., Kawaguchi, T., Peterson, B. M. \& Vestergaard, M. 2006, A\&A, 456, 75 


\section{Approach 1}

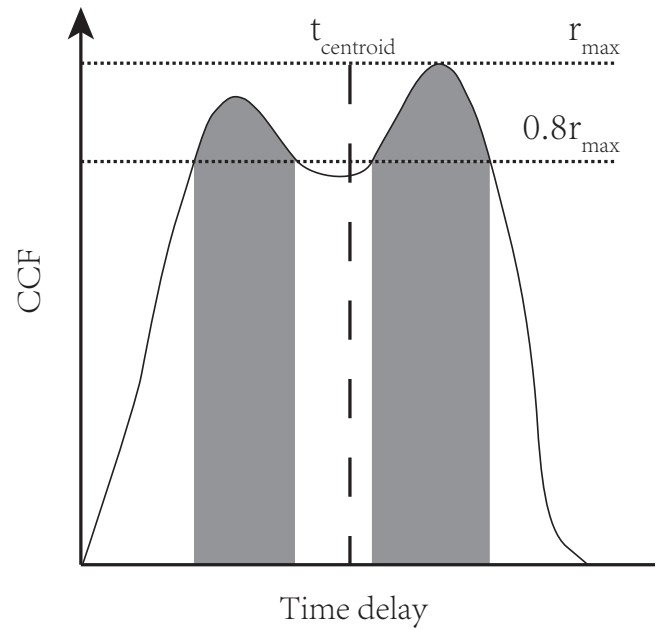

Approach 2

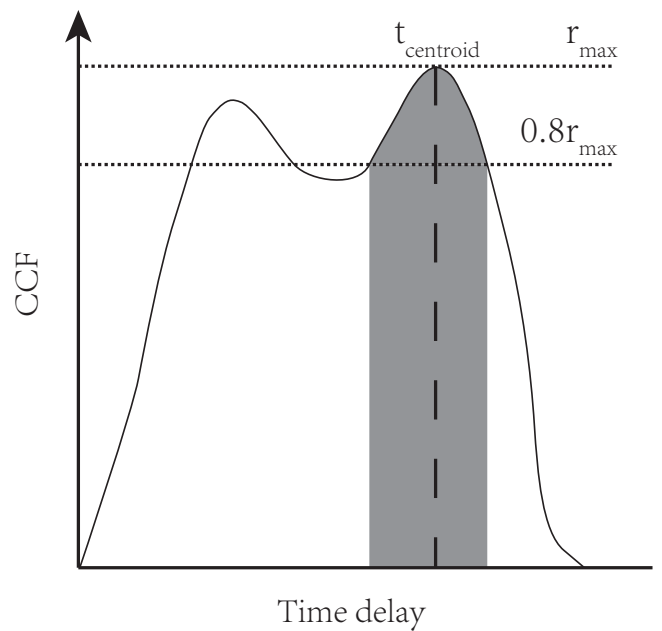

Figure 9. Two approaches to calculate centroid time lag. We adopted Approach 1 in our analysis.

Czerny, B., Hryniewicz, K., Maity, I. et al. 2013, A\&A, 556, 97

Czerny, B. \& Elvis, M. 1987, ApJ, 321, 305

Davis, S. W. \& Laor, A. 2011, ApJ, 728, 98

Denney, K. D., Bentz, M. C., Peterson, B. M. et al. 2006, ApJ, 653, 152

Denney, K. D. et al. 2009, ApJ, 702, 1353

Denney, K. D., Peterson, M. C., Pogge, R. W. et al. 2010, ApJ, 721, 715

Dietrich, M., Kollatschny, W., Peterson, B. M., et al. 1993, ApJ, 408, 416

Dietrich, M., Peterson, B. M., Albrecht, P. et al. 1998, ApJS, 115, 185

Dietrich, M., Peterson, B. M., Grier, C. J. et al. 2012, ApJ, 757, 53

Du, P., Hu, C., Lu, K.-X., et al. 2014, ApJ, 782, 45 (Paper I)

Du, P., Lu, K.-X., Hu, C., et al. 2015, ApJ, 820, 27 (Paper VI)

Du, P., Hu, C., Lu, K.-X., et al. 2016a, ApJ, 806, 22 (Paper IV)

Du, P., Wang, J.-M., Hu, C., Ho, L. C., Li, Y.-R. \& Bai, J.-M., 2016b, ApJL, 818, L14

Fischer, T. C., Crenshaw, D. M., Kraemer, S. B., et al. 2014, ApJ, 785, 25

Gammie, C. F. 1998, MNRAS, 297, 929

Giannuzzo, M. Z., Mignoli, M., Stirpe, G. M. \& Comastri, A. 1998, A\&A, 330,894

Giannuzzo, M. Z. \& Stirpe, G. M. 1996, A\&A, 314, 419

Goad, M. R. \& Korista, K. T. 2014, MNRAS, 444, 43

Greene, J. \& Ho, L. C. 2006, ApJ, 636, 56

Greene, J. \& Ho, L. C. 2007, ApJ, 670, 92

Grier, C. J., Peterson, B. M., Pogge, R. W. et al. 2012, ApJ, 755, 60

Ho, L. C. 2002, ApJ, 564, 120

Ho, L. C. 2008, ARA\&A, 46, 475

Ho, L. C., \& Kim, M. 2016, ApJ, in press (arXiv:1603.00057)

Horne, K., Korista, K. T. \& Goad, M. G. 2003, MNRAS, 339, 367

Hoyle, F. \& Burbidge, R, 1966, Nature, 210, 1346

Hu, C., Du, P., Lu, K.-X. et al. 2015, ApJ, 804, 138 (Paper III)

Hu, C., Wang, J.-M. \& Ho, L. C. et al. 2008a, ApJ, 687, 78

Hu, C., Wang, J.-M. \& Ho, L. C. et al. 2008b, ApJ, 683, L115

Jiang, Y.-F., Stone, J. M. \& Davis, S. W. 2014, ApJ, 796, 106

Kaspi, S., Maoz, D., Netzer, H. et al. 2005, ApJ, 629, 61

Kaspi, S., Smith, P. S., Netzer, H. et al. 2000, ApJ, 533, 631

Kilerci Eser, E., Vestergaard, M., Peterson, B. M. et al. 2015, ApJ, 801, 8

Klimek, E. S., Gaskell, C. M. \& Hedrick, C. H. 2004, ApJ, 609, 69

Kishimoto M., Antonucci R., Blaes O. et al. 2008, Nature, 454, 492

Koratkar, A. P. \& Gaskell, C. M. 1991, ApJ, 370, L61

La Franca, F., Bianchi, S. \& Ponti, G. et al. 2014, ApJ, 787, L12

Laor, A. \& Netzer, H. 1989, MNRAS, 238, 897

Li, G.-X., Yuan, Y.-F. \& Cao, X., 2010, ApJ, 715, 623

Li, Y.-R., Wang, J.-M., Ho, L. C., Du, P. \& Bai, J.-M. 2013, ApJ, 779, 110

Longair, M. S. \& Scheuer, P. A. G. 1967, Nature, 215, 919

Maoz, D. \& Netzer, H. 1989, MNRAS, 236, 21

Maoz, D., Netzer, H., Leibowitz, E., et al. 1990, ApJ, 351, 75

Maoz, D., Netzer, H., Mazeh, T. et al. 1991, ApJ, 367, 493

Marziani, P. \& Sulentic, J. 2014, MNRAS, 442, 1211
Mineshige, S., Kawaguchi, T., Takeuchim M., \& Hayashida, K. 2000, PASJ, 52,499

Narayan, R. \& Yi, I. 1994, ApJ, 428, L13

Netzer, H., Heller, A., Loinger, F. et al., 1996, MNRAS, 279, 429

Netzer, H., Shemmer, O., Maiolino, R. et al. 2004, ApJ, 614, 558

Osterbrock, D. E. \& Pogge, R. W. 1987, ApJ, 323, 108

Pancoast, A., Brewer, B. J., Treu, T. et al. 2014, MNRAS, 445, 3073

Peterson, B. M., Balonek, T. J., Barker, E. S. et al. 1991, ApJ, 368, 119

Peterson, B. M. 1993, PASP, 105, 247

Peterson, B. M., Ferrarese, L., Gilbert, K. M. et al. 2004, ApJ, 613, 682

Peterson, B. M., Grier, C. J., Horne, K. et al. 2014, ApJ, 795, 149

Peterson, B. M., Wanders, I., Bertram, R. et al. 1998, ApJ, 501, 82

Risaliti, R. \& Lusso, E. 2015, arXiv:1505.07118

Sadowski, A. 2009, ApJS, 183, 171

Sadowski, A., Abramowicz, M. \& Bursa, M. 2011, A\&A, 527, A17

Sadowski, A., Narayan, R., McKinney, J. C., \& Tchekhovskoy, A. 2014, MNRAS, 439, 503

Sadowski, A., Narayan, R., Tchekhovskoy, A. et al. 2015, MNRAS, 447, 49

Sandage, A. 1965, ApJ, 141, 1560

Schmidt, M. 1968, ApJ, 151, 393

Shakura, N. I., \& Sunyaev, R. A. 1973, A\&A, 24, 337

Shen, Y. \& Ho, L. C. 2014, Nature, 513, 210

Shen, Y, Brandt, W. N., Dawson, K. S. et al. 2015, ApJS, 216, 4

Shen, Y., Horne, K., Grier, C. J., et al. 2015b, arXiv:1510.02802

Shen, Y., Richards, G. T., Strauss, M. A., et al. 2011, ApJS, 194, 45

Sulentic, J., Marziani, P. \& Dultzin-Hacyan, D. 2000, ARA\&A, 38, 521

Sun, W.-H. \& Malkan, M. A. 1989, ApJ, 346, 68

Szuszkiewicz, E., Malkan, M. A., \& Abramowicz, M. A. 1996, ApJ, 458, 474

Tremaine, S., Gebhardt, K., Bender, R., et al. 2002, ApJ, 574, 740

Vestergaard, M. \& Peterson, B. M. 2006, ApJ, 641, 689

van Groningen, E., \& Wanders, I. 1992, PASP, 104, 700

Wanders, I., van Groningen, E., Alloin, D. et al. 1993, A\&A, 269, 39

Wandel, A. \& Petrosian, V. 1988, ApJ, 329, L11

Wang, J.-M., Watarai, K. \& Mineshige, S. 2004, ApJ, 607, L107

Wang, J.-M., Du, P., Hu, C., et al. 2014a, ApJ, 793, 108 (Paper II)

Wang, J.-M., Du, P., Valls-Gabaud, D., Hu, C., \& Netzer, H. 2013,

Phys. Rev. Lett., 110, 081301

Wang, J.-M., Qiu, J., Du, P. \& Ho, L. C. 2014b, ApJ, 797, 65

Wang, J.-M., Du, P., Li, Y.-R., Ho, L. C., Hu, C. \& Bai, J.-M. 2014c, ApJ, 792, L13

Wang, J.-M., \& Netzer, H. 2003, A\&A, 398, 327

Wang, J.-M., Szuszkiewicz, E., Lu, F.-J., \& Zhou, Y.-Y. 1999, ApJ, 522, 839

Wang, J.-M., \& Zhou, Y.-Y. 1999, ApJ, 516, 420

Watson, D., Denney, K. D., Vestergaard, M., \& Davis, T. M. 2011, ApJ, 740, L49

Woo, J.-H., Yoon, Y., Park, S., Park, D. \& Kim, S. C. 2015, ApJ, 801, 38

Zhao, G.-B., Crittenden, R. G., Pogosian, L. \& Zhang, X. 2012,

Phys. Rev. Lett., 109, 1301 